Article

\title{
On Schwarzschild's Interior Solution and Perfect Fluid Star Model
}

\author{
Elisabetta Barletta ${ }^{1}$, Sorin Dragomir 1,* and Francesco Esposito ${ }^{2}$ \\ 1 Dipartimento di Matematica, Informatica, ed Economia, Università degli Studi della Basilicata, \\ 85100 Potenza, Italy; elisabetta.barletta@unibas.it \\ 2 Dipartimento di Matematica e Fisica Ennio De Giorgi, Università del Salento, 73100 Lecce, Italy; \\ francesco.esposito@unisalento.it \\ * Correspondence: sorin.dragomir@unibas.it; Tel.: +39-0971-205843
}

Received: 17 September 2020; Accepted: 30 September 2020 ; Published: 13 October 2020

\begin{abstract}
We solve the boundary value problem for Einstein's gravitational field equations in the presence of matter in the form of an incompressible perfect fluid of density $\rho$ and pressure field $p(r)$ located in a ball $r \leq r_{0}$. We find a 1-parameter family of time-independent and radially symmetric solutions $\left\{\left(g_{a}, \rho_{a}, p_{a}\right):-2 m<a<a_{1}\right\}$ satisfying the boundary conditions $g=g_{S}$ and $p=0$ on $r=r_{0}$, where $g_{S}$ is the exterior Schwarzschild solution (solving the gravitational field equations for a point mass $M$ concentrated at $r=0$ ) and containing (for $a=0$ ) the interior Schwarzschild solution, i.e., the classical perfect fluid star model. We show that Schwarzschild's requirement $r_{0}>9 \kappa M /\left(4 c^{2}\right)$ identifies the "physical" (i.e., such that $p_{a}(r) \geq 0$ and $p_{a}(r)$ is bounded in $\left.0 \leq r \leq r_{0}\right)$ solutions $\left\{p_{a}: a \in U_{0}\right\}$ for some neighbourhood $U_{0} \subset(-2 m,+\infty)$ of $a=0$. For every star model $\left\{g_{a}: a_{0}<a<a_{1}\right\}$, we compute the volume $V(a)$ of the region $r \leq r_{0}$ in terms of abelian integrals of the first, second, and third kind in Legendre form.
\end{abstract}

Keywords: Schwarzschild's solution; boundary value problem; abelian integral

\section{A Boundary Value Problem}

The interior Schwarzschild solution (cf. K. Schwarzschild, [1])

$$
\begin{gathered}
g=\left[\frac{3}{2} \sqrt{1-\left(\frac{r_{0}}{\hat{R}}\right)^{2}}-\frac{1}{2} \sqrt{1-\left(\frac{r}{\hat{R}}\right)^{2}}\right]^{2} c^{2} d t^{2}-\frac{d r^{2}}{1-\left(\frac{r}{\hat{R}}\right)^{2}}-r^{2}\left(d \theta^{2}+\sin ^{2} \theta d \varphi^{2}\right) \\
r \leq r_{0}, \quad \hat{R}^{2}=\frac{3 c^{2}}{8 \pi \kappa \rho},
\end{gathered}
$$

provides a simple model of a star, shaped as a homogeneous sphere of incompressible fluid with radius $r_{0}$. The Lorentzian metric (1) is a time-independent and radially symmetric solution to the boundary value problem for gravitational field equations

$$
\begin{gathered}
R^{\alpha \gamma}-\frac{1}{2} g^{\alpha \gamma} R=-\frac{8 \pi \kappa}{c^{2}} T^{\alpha \gamma} \text { in } r<r_{0}, \\
g^{\alpha \gamma}=\left(g_{S}\right)^{\alpha \gamma} \text { and } p=0 \text { on } r=r_{0},
\end{gathered}
$$

for nonempty space, whose matter and energy content is described by the energy-momentum tensor

$$
T_{\alpha \beta}=\rho u_{\alpha} u_{\beta}+\frac{p}{c^{2}}\left(u_{\alpha} u_{\beta}-g_{\alpha \beta}\right)
$$


where $\rho$ and $p$ are the density and pressure fields, respectively, and $g_{S}$ is the exterior Schwarzschild solution, i.e.,

$$
\begin{gathered}
g_{S}=\left(1-\frac{2 m}{r}\right) c^{2} d t^{2}-\left(1-\frac{2 m}{r}\right)^{-1} d r^{2}-r^{2}\left(d \theta^{2}+\sin ^{2} \theta d \varphi^{2}\right), \\
m=\frac{\kappa M}{c^{2}}, \quad r \geq r_{0} .
\end{gathered}
$$

Lorentzian metric $g_{S}$ is only defined in the region of $r>2 m$. In particular, it must be

$$
r_{0}>2 m \text {. }
$$

Solutions to (2) and (3) are looked for in the form

$$
g=e^{v(r)} c^{2} d t^{2}-\left[e^{\lambda(r)} d r^{2}+r^{2}\left(d \theta^{2}+\sin ^{2} \theta d \varphi^{2}\right)\right]
$$

where the unknown functions $v(r)$ and $\lambda(r)$ are determined from the field equations under the additional assumption that the fluid matter is at rest, i.e., the components of the velocity four vector $u^{\alpha}$ are $\left(u^{0}, 0,0,0\right)$ with $g_{00}\left(u^{0}\right)^{2}=1$. If we set $u_{\alpha}=g_{\alpha \beta} u^{\beta}$ then

$$
u_{0}=g_{0 \beta} u^{\beta}=g_{00} u^{0}=\sqrt{g_{00}}, \quad u_{i}=0,
$$

hence, $T_{\alpha \beta}$ becomes

$$
\left[T_{\alpha \beta}\right]=\left(\begin{array}{cccc}
\rho e^{v(r)} & 0 & 0 & 0 \\
0 & \frac{p}{c^{2}} e^{\lambda(r)} & 0 & 0 \\
0 & 0 & \frac{p}{c^{2}} r^{2} & 0 \\
0 & 0 & 0 & \frac{p}{c^{2}} r^{2} \sin ^{2} \theta
\end{array}\right)
$$

(the energy-momentum tensor for the perfect fluid at rest). The nonzero components of the Ricci tensor of the metric (7) are

$$
\begin{gathered}
R_{00}=e^{v-\lambda}\left(-\frac{v^{\prime \prime}}{2}+\frac{1}{4} \lambda^{\prime} v^{\prime}-\frac{1}{4} v^{\prime 2}-\frac{v^{\prime}}{r}\right), \\
R_{11}=\frac{1}{2} v^{\prime \prime}-\frac{1}{4} v^{\prime} \lambda^{\prime}+\frac{1}{4} v^{\prime 2}-\frac{\lambda^{\prime}}{r} \\
R_{22}=e^{-\lambda}\left(1+\frac{1}{2} r v^{\prime}-\frac{1}{2} r \lambda^{\prime}\right)-1, \quad R_{33}=R_{22} \sin ^{2} \theta .
\end{gathered}
$$

Then, scalar curvature $R=g^{\alpha \beta} R_{\alpha \beta}$ is

$$
R=-e^{-\lambda}\left[v^{\prime \prime}-\frac{1}{2} v^{\prime} \lambda^{\prime}+\frac{1}{2} v^{\prime 2}+2\left(\frac{v^{\prime}-\lambda^{\prime}}{2}\right)+\frac{2}{r^{2}}\right]+\frac{2}{r^{2}} .
$$

Substitution from (8) -(12) into (2) turns (2) and (3) into the boundary value problem

$$
\begin{gathered}
C \rho=e^{-\lambda}\left(\frac{1}{r^{2}}-\frac{\lambda^{\prime}}{r}\right)-\frac{1}{r^{2}}, \\
C \frac{p}{c^{2}}=\frac{1}{r^{2}}-e^{-\lambda}\left(\frac{1}{r^{2}}+\frac{v^{\prime}}{r}\right),
\end{gathered}
$$




$$
\begin{gathered}
C \frac{p}{c^{2}}=e^{-\lambda}\left[\frac{1}{4} v^{\prime} \lambda^{\prime}-\frac{1}{4} \lambda^{\prime 2}-\frac{1}{2} v^{\prime \prime}-\frac{1}{2}\left(\frac{v^{\prime}-\lambda^{\prime}}{r}\right)\right] \\
v\left(r_{0}\right)=\log \left(1-\frac{2 m}{r_{0}}\right), \lambda\left(r_{0}\right)=-\log \left(1-\frac{2 m}{r_{0}}\right), \quad p\left(r_{0}\right)=0
\end{gathered}
$$

where $C=-8 \pi \kappa / c^{2}$. There are but three independent field Equations (13)-(15), for Equations (2) with $(\alpha, \gamma) \in\{(2,2),(3,3)\}$ coincide. So, (13)-(15) is a system of ODEs with four unknowns $(\nu, \lambda, \rho, p)$ and a physically reasonable solution should satisfy $\rho(r)>0$ and $p(r) \geq 0$. The addition of (13) and (14)

$$
-C\left(\rho+\frac{p}{c^{2}}\right)=\frac{e^{-\lambda}}{r}\left(v^{\prime}+\lambda^{\prime}\right)
$$

yields $v^{\prime}+\lambda^{\prime}>0$ [for any physically reasonable solution $(\nu, \lambda, \rho, p)$ ]. Let us eliminate $p$ between (14) and (15), respectively differentiate (14), so that to obtain

$$
\begin{gathered}
\frac{e^{\lambda}}{r^{2}}=\frac{1}{4} v^{\prime} \lambda^{\prime}-\frac{1}{4} v^{\prime 2}-\frac{1}{2} v^{\prime \prime}+\frac{v^{\prime}+\lambda^{\prime}}{2 r}+\frac{1}{r^{2}} \\
C \frac{p^{\prime}(r)}{c^{2}}=-\frac{2}{r^{3}}+e^{-\lambda}\left(\frac{\lambda^{\prime}}{r^{2}}+\frac{\lambda^{\prime} v^{\prime}}{r}+\frac{2}{r^{3}}+\frac{v^{\prime}}{r^{2}}-\frac{v^{\prime \prime}}{r}\right) .
\end{gathered}
$$

Elimination of $v^{\prime \prime}$ among (18) and (19) then leads to

$$
C \frac{p^{\prime}(r)}{c^{2}}=e^{-\lambda} \frac{v^{\prime}}{2 r}\left(\lambda^{\prime}+v^{\prime}\right) .
$$

Lastly, let us eliminate $\lambda$ between (17) and (20) so that to obtain

$$
\frac{p^{\prime}(r)}{c^{2}}=-\frac{1}{2} v^{\prime}(r)\left[\rho(r)+\frac{p(r)}{c^{2}}\right],
$$

which readily determines $\nu$, provided that $\rho$ and $p$ are known. The main result in [1] is that ODEs system (13)-(15) may be integrated under the additional assumption that the fluid is incompressible, i.e., $\rho(r)=\rho=$ constant. The assumption that $\rho$ is constant is justified by its advantages in the mathematical handling of Equations (13)-(15). Indeed, if $\rho \in(0,+\infty)$, then (13) readily furnishes

$$
e^{-\lambda}=1+C \rho \frac{r^{2}}{3}+\frac{a}{r}
$$

where $a \in \mathbb{R}$ is a constant of integration. If $a \in \mathbb{R} \backslash\{0\}$ then $\lambda(0)=-\infty$ hence [by (7)] det $\left[g_{\alpha \beta}\right]=0$ at $r=0$ i.e., the space-time described by $g$ is singular on the 2-surface $r=0$, at least from the nineteenth-century perspective adopted in [2] (pp. 1-3). To derive a solution without singularities of the sort, K. Schwarzschild chose (cf. [1]) $a=0$ (and then $\lambda$ is readily determined from (22)). It is our purpose in the present paper to determine the remaining (a fortiori unbounded) solutions $(\nu, \lambda, \rho, p)$ to the boundary value problem (13)-(16) corresponding to choices $a \in \mathbb{R} \backslash\{0\}$. Other interior Schwarzschild-type solutions were determined by P.S. Florides (cf. [3]), A.L. Mehra (cf. [4]), N.K. Kofinti (cf. [5]), and O. Gron (cf. [6], further generalizing the work in [3]). Let us set

$$
\hat{R}^{2}:=-\frac{3}{C \rho}=\frac{3 c^{2}}{8 \pi \kappa \rho}
$$

so that (22) reads

$$
-g_{11}=e^{\lambda}=\left(1-\frac{r^{2}}{\hat{R}^{2}}+\frac{a}{r}\right)^{-1} .
$$


Next, let us integrate (21) (under the assumption that $\rho$ is constant) to obtain

$$
\frac{p}{c^{2}}+\rho=-\frac{D}{C} e^{-v / 2}
$$

where $D \in \mathbb{R}$ is a constant of integration. Moreover, we eliminate $p / c^{2}+\rho$ between (24) and (17)

$$
\frac{e^{-\lambda}}{r}\left(v^{\prime}+\lambda^{\prime}\right)=D e^{-v / 2}
$$

which may be written

$$
r D e^{-v / 2}=e^{-\lambda} v^{\prime}-\left(e^{-\lambda}\right)^{\prime}
$$

Let us replace $e^{-\lambda}$ from (23) to obtain the following ODE with the unknown $v$

$$
r D e^{-v / 2}=\left(1-\frac{r^{2}}{\hat{R}^{2}}+\frac{a}{r}\right) v^{\prime}+\frac{2 r}{\hat{R}^{2}}+\frac{a}{r^{2}} .
$$

By a change of dependent variable $\gamma(r)=e^{v(r) / 2}$, Equation (26) may be written

$$
\left(1-\frac{r^{2}}{\hat{R}^{2}}+\frac{a}{r}\right) \gamma^{\prime}+\left(\frac{r}{\hat{R}^{2}}+\frac{a}{2 r^{2}}\right) \gamma=\frac{1}{2} r D .
$$

To solve the family of ODEs (27), one should integrate the associated homogenous equation

$$
\left(1-\frac{r^{2}}{\hat{R}^{2}}+\frac{a}{r}\right) u^{\prime}+\left(\frac{r}{\hat{R}^{2}}+\frac{a}{2 r^{2}}\right) u=0
$$

or

$$
u^{\prime}-U_{a}(r) u=0, \quad U_{a}(r):=\frac{2 r^{3}+a \hat{R}^{2}}{2 r\left(r^{3}-\hat{R}^{2} r-a \hat{R}^{2}\right)}
$$

i.e.,

$$
\log u=\int U_{a}(r) d r+\alpha, \quad \alpha \in \mathbb{R} .
$$

For every $\lambda \in \mathbb{C}$ and $n \in \mathbb{N}$, we adopt customary notation $\sqrt[n]{\lambda}=|\lambda|^{1 / n} \exp [i \arg (\lambda) / n]$, where $\arg : \mathbb{C} \rightarrow[0,2 \pi)$. Let $I_{3}=\left\{\zeta \in \mathbb{C}: \zeta^{3}=1\right\}=\left\{\omega_{0}, \omega_{1}, \omega_{2}\right\}$. To compute the integral in (29), let us consider the polynomial $P_{a}(r)=r^{3}-\hat{R}^{2} r-a \hat{R}^{2} \in \mathbb{R}[r]$ and set

$$
\left\{\lambda_{1}, \lambda_{2}\right\}:=\left\{\frac{a \hat{R}^{2}}{2} \pm \sqrt{\frac{a^{2} \hat{R}^{4}}{4}-\frac{\hat{R}^{6}}{27}}\right\} \subset \mathbb{C} .
$$

We need to distinguish among the following cases: (I) $a^{2}>\frac{4}{27} \hat{R}^{2}$, (II) $a^{2}<\frac{4}{27} \hat{R}^{2}$, and (III) $a^{2}=\frac{4}{27} \hat{R}^{2}$. In Case (I), one has $a \neq 0$ and $\left\{\lambda_{1}, \lambda_{2}\right\} \subset \mathbb{R}$, while $a>0 \Longrightarrow \lambda_{1}>0, \lambda_{2}>0$ and $a<0 \Longrightarrow \lambda_{1}<0$, $\lambda_{2}<0$. Moreover, set $X_{a}=\left\{x \in \mathbb{C}: P_{a}(x)=0\right\}$ is

$$
X_{a}=\left\{\begin{array}{l}
\left\{\omega_{k} \sqrt[3]{\lambda_{1}}+\omega_{\ell} \sqrt[3]{\lambda_{k}}:(k, \ell) \in\{(0,0),(1,2),(2,1)\} \quad \text { if } a>0\right. \\
\left\{\omega_{k} \sqrt[3]{\lambda_{1}}+\omega_{\ell} \sqrt[3]{\lambda_{k}}:(k, \ell) \in\{(0,2),(1,1),(2,0)\} \quad \text { if } a<0\right.
\end{array}\right.
$$

where $\omega_{k}=\exp (2 k \pi i / 3) \in I_{3}$. Consequently, one has the decomposition

$$
U_{a}=\frac{1}{2}\left[-\frac{1}{r}+\frac{1}{r-\sigma}+\frac{2 r+\sigma}{r^{2}+\sigma r+\tau}\right]
$$


where $\sigma:=\sqrt[3]{\lambda_{1}}+\sqrt[3]{\lambda_{2}}$ and $\tau:=\left(\sqrt[3]{\lambda_{1}}\right)^{2}-\sqrt[3]{\lambda_{1}} \sqrt[3]{\lambda_{2}}+\left(\sqrt[3]{\lambda_{2}}\right)^{2}$ (keeping in mind that $\sigma \in \mathbb{R}$ and $\tau>0)$. Next,

$$
\int U_{a}(r) d r=\frac{1}{2} \log \frac{|r-\sigma|\left(r^{2}+\sigma r+\tau\right)}{r}+\alpha=\frac{1}{2} \log \frac{\left|r^{3}-\hat{R}^{2} r-a \hat{R}^{2}\right|}{r}+\alpha
$$

Hence,

$$
u(r)=u_{a}(r):=B \sqrt{\frac{\left|r^{3}-\hat{R}^{2} r-a \hat{R}^{2}\right|}{r}},
$$

where $B \in \mathbb{R}$ is a constant of integration (the general solution to (28)). For $a=0$, the solution obtained in [1] [i.e., the general solution to homogenous equation $\left(1-\frac{r^{2}}{\hat{R}^{2}}\right) u^{\prime}(r)+\frac{r}{\hat{R}^{2}} u(r)=0$ ] is

$$
u=B\left(1-\frac{r^{2}}{\hat{R}^{2}}\right)^{1 / 2}, \quad B \in \mathbb{R},
$$

[coinciding with $u_{0}$ as given by (30)]. In Case (II), one has $\lambda_{1}, \lambda_{2} \in \mathbb{C} \backslash \mathbb{R}, \lambda_{2}=\bar{\lambda}_{1}$, and set $X_{a}$ is

$$
X_{a}=\left\{\omega_{k} \sqrt[3]{\lambda_{1}}+\omega_{\ell} \sqrt[3]{\lambda_{2}}:(k, \ell) \in\{(0,2),(1,1),(2,0)\}\right\}=\left\{x_{0}, x_{1}, x_{2}\right\} \subset \mathbb{R}
$$

yielding the decomposition

$$
\begin{gathered}
U_{a}(r)=\frac{1}{2}\left[-\frac{1}{r}+\frac{3 x_{0}^{2}-\hat{R}^{2}}{\left(x_{0}-x_{1}\right)\left(x_{0}-x_{2}\right)\left(r-x_{0}\right)}+\frac{\hat{R}^{2}-3 x_{1}^{2}}{\left(x_{0}-x_{1}\right)\left(x_{1}-x_{2}\right)\left(r-x_{1}\right)}\right. \\
\left.+\frac{3 x_{2}^{2}-\hat{R}^{2}}{\left(x_{0}-x_{2}\right)\left(x_{1}-x_{2}\right)\left(r-x_{2}\right)}\right]=\frac{1}{2}\left[-\frac{1}{r}+\frac{1}{r-x_{0}}+\frac{1}{r-x_{1}}+\frac{1}{r-x_{2}}\right]
\end{gathered}
$$

so that once again

$$
\int U_{a}(r) d r=\log \sqrt{\frac{\left|P_{a}(r)\right|}{r}}+\alpha
$$

leading to (30). In Case (III),

$$
a \in\left\{ \pm \frac{2 \hat{R}}{3 \sqrt{3}}\right\} \Longrightarrow X_{a}=\left\{ \pm \frac{\hat{R}}{\sqrt{3}}\right\} \cup\left\{\frac{2 \hat{R}}{\sqrt{3}}\right\}
$$

and $P_{a}$ decomposes as $P_{a}=(r \pm \hat{R} \sqrt{3})^{2}\left(r \mp \frac{2 \hat{R}}{\sqrt{3}}\right)$ leading to (30), i.e.,

$$
u_{a}(r)=B \sqrt{\frac{1}{r}\left[r^{3}-\hat{R}^{2} r \mp \frac{2 \hat{R}^{3}}{3 \sqrt{3}}\right]} .
$$

The associated inhomogeneous equation (i.e., (27) with $a=0$ )

$$
\left(1-\frac{r^{2}}{\hat{R}^{2}}\right) \gamma^{\prime}(r)+\frac{r}{\hat{R}^{2}} \gamma=\frac{1}{2} r D
$$

admits the obvious (constant) solution $\gamma_{0}=\frac{1}{2} D \hat{R}^{2}$; hence, the general solution to (32) is

$$
\frac{1}{2} D \hat{R}^{2}-B\left(1-\frac{r^{2}}{\hat{R}^{2}}\right)^{1 / 2} .
$$


The determination of a particular solution to (27) is a considerably more difficult problem, involving hyperelliptic integrals. Equation (27) reads

$$
\gamma^{\prime}-\frac{2 r^{3}+a \hat{R}^{3}}{2 r P_{a}(r)} \gamma=-\frac{D \hat{R}^{2} r^{2}}{2 P_{a}(r)}
$$

or $\gamma^{\prime}-U_{a}(r) \gamma=-\frac{D \hat{R}^{2} r^{2}}{2 P_{a}(r)}$; hence, (by (30) with $B=1$ )

$$
\gamma_{a}(r)=-\frac{D \hat{R}^{2}}{2} \sqrt{\frac{\left|P_{a}(r)\right|}{r}} \int \frac{r^{5 / 2} d r}{P_{a}(r) \sqrt{\left|P_{a}(r)\right|}}
$$

is a particular solution to inhomogeneous Equation (27). Hence, the general solution to (27) is

$$
e^{v / 2}=\gamma_{a}(r)-B \hat{R}\left(\frac{\left|P_{a}(r)\right|}{r}\right)^{1 / 2}, B \in \mathbb{R},
$$

so that

$$
g_{00}=e^{v}=\left[\gamma_{a}(r)-B \hat{R}\left(\frac{\left|P_{a}(r)\right|}{r}\right)^{1 / 2}\right]^{2} .
$$

Let $g_{a}$ be the metric tensor defined by (7) with $g_{00}=e^{v}$ and $g_{11}=-e^{\lambda}$, respectively given by (23) and (35), i.e.,

$$
g_{a}=\left[\gamma_{a}(r)-B \hat{R} \sqrt{\frac{\left|P_{a}(r)\right|}{r}}\right]^{2} c^{2} d t^{2}-\left(1-\frac{r^{2}}{\hat{R}^{2}}+\frac{a}{r}\right)^{-1} d r^{2}-r^{2}\left(d \theta^{2}+\sin ^{2} \theta d \varphi^{2}\right)
$$

$\left(0<r \leq r_{0}\right)$. Let us set $A=\frac{1}{2} D \hat{R}^{2}$ and $J_{a}(r)=\int \frac{r^{5 / 2} d r}{\left|P_{a}(r)\right|^{3 / 2}}$, so that

$$
\gamma_{a}(r)=-A \operatorname{sign}\left[P_{a}(r)\right] \sqrt{\frac{\left|P_{a}(r)\right|}{r}} J_{a}(r)
$$

and then

$$
g_{00}=\frac{\left|P_{a}(r)\right|}{r}\left[A \operatorname{sign}\left[P_{a}(r)\right] J_{a}(r)+B \hat{R}\right]^{2} .
$$

As is customary, the constants of integration $A, B \in \mathbb{R}$ are determined by the boundary conditions that the coefficients of the Lorentzian metrics (36) and (5) coincide on $r=r_{0}$, and $p\left(r_{0}\right)=0$ ( $p$ vanishes at the boundary, so that it matches continuously with the zero pressure at the exterior of the fluid star). The identification of $g_{11}$ coefficients (in (36) and (5) at $r=r_{0}$ )

$$
1-\frac{r_{0}^{2}}{\hat{R}^{2}}+\frac{a}{r_{0}}=1-\frac{2 m}{r_{0}}
$$

yields

$$
a=\frac{r_{0}^{3}}{\hat{R}^{2}}-2 m
$$

This is equivalent to $\hat{R}^{2}=r_{0}^{3} /(a+2 m)$ and yields

$$
a>-2 m .
$$


By taking into account $\hat{R}^{2}=3 c^{2} /(8 \pi \kappa \rho)$ and $m=\kappa M / c^{2}$, we may rewrite (38) as

$$
M=\frac{4 \pi r_{0}^{3}}{3} \rho-\frac{c^{2}}{2 \kappa} a
$$

and density $\rho$ is determined [under the requirement of regularity (i.e., looking for a bounded solution $\lambda) a=0$ (as in [1]), and the boundary condition (40) leads to

$$
M=4 \pi r_{0}^{3} \rho / 3
$$

(determining $\rho)$ ]. We are left with the determination of constants $A$ and $B$ in (36). To this end, let us return to (24) and substitute $e^{v / 2}$ from (35) so that to obtain

$$
C\left(\frac{p}{c^{2}}+\rho\right)=-D\left[\gamma_{a}-B \hat{R} \sqrt{\frac{\left|P_{a}(r)\right|}{r}}\right]^{-1}
$$

which evaluated at $r=r_{0}$ gives (by the boundary condition $p\left(r_{0}\right)=0$ )

$$
C \rho=-D\left[\gamma_{a}\left(r_{0}\right)-B \hat{R} \sqrt{\frac{\left|P_{a}\left(r_{0}\right)\right|}{r_{0}}}\right]^{-1}
$$

or (by $D=2 A / \hat{R}^{2}$ and $\hat{R}^{2}=-3 / C \rho$ together with (37))

$$
\frac{2 A}{A \operatorname{sign}\left[P_{a}\left(r_{0}\right)\right] J_{a}\left(r_{0}\right)+B \hat{R}}=-3 \sqrt{\frac{\left|P_{a}\left(r_{0}\right)\right|}{r_{0}}}
$$

or

$$
A=-\frac{3 B \hat{R} \sqrt{\frac{\left|P_{a}\left(r_{0}\right)\right|}{r_{0}}}}{2+3 \operatorname{sign}\left[P_{a}\left(r_{0}\right)\right] \sqrt{\frac{\left|P_{a}\left(r_{0}\right)\right|}{r_{0}}} J_{a}\left(r_{0}\right)} .
$$

Moreover, by identifying the $g_{00}$ coefficients (of Lorentzian metrics (36) and (5))at $r=r_{0}$

$$
\frac{\left|P_{a}\left(r_{0}\right)\right|}{r_{0}}\left[A \operatorname{sign}\left[P_{a}\left(r_{0}\right)\right] J_{a}\left(r_{0}\right)+B \hat{R}\right]^{2}=1-\frac{2 m}{r_{0}}
$$

or (by (72))

$$
\frac{4 B^{2} \hat{R}^{2} \frac{\left|P_{a}\left(r_{0}\right)\right|}{r_{0}}}{\left[2+3 \operatorname{sign}\left[P_{a}\left(r_{0}\right)\right] \sqrt{\frac{\left|P_{a}\left(r_{0}\right)\right|}{r_{0}}} J_{a}\left(r_{0}\right)\right]^{2}}=1-\frac{2 m}{r_{0}}
$$

yielding

$$
A=-\frac{3 \epsilon_{a}}{2}\left(1-\frac{2 m}{r_{0}}\right)^{1 / 2}
$$




$$
B=\frac{1}{2 \hat{R}}\left(1-\frac{2 m}{r_{0}}\right)^{1 / 2} \frac{\left|2+3 \operatorname{sign}\left[P_{a}\left(r_{0}\right)\right] \sqrt{\frac{\left|P_{a}\left(r_{0}\right)\right|}{r_{0}}} J_{a}\left(r_{0}\right)\right|}{\sqrt{\frac{\left|P_{a}\left(r_{0}\right)\right|}{r_{0}}}},
$$

where $\epsilon_{a}=\operatorname{sign}\left\{2+3 \operatorname{sign}\left[P_{a}\left(r_{0}\right)\right] \sqrt{\frac{\left|P_{a}\left(r_{0}\right)\right|}{r_{0}}}\right\}$. Let us substitute from (43)-(74) into (36)-(37) so that to obtain

$$
\begin{gathered}
g_{a}=g_{00} c^{2} d t^{2}-\left(1-\frac{r^{2}}{\hat{R}^{2}}+\frac{a}{r}\right)^{-1} d r^{2}-r^{2}\left(d \theta^{2}+\sin ^{2} \theta d \varphi^{2}\right), \\
g_{00}=\frac{1}{4}\left(1-\frac{2 m}{r_{0}}\right) \frac{\left|P_{a}(r)\right|}{r}\left[F_{a}(r)-F_{a}\left(r_{0}\right)-2 \sqrt{\frac{r_{0}}{\left|P_{a}\left(r_{0}\right)\right|}}\right]^{2}, \\
F_{a}(r)=3 \operatorname{sign}\left[P_{a}(r)\right] J_{a}(r), \quad 1-\frac{2 m}{r_{0}}=1-\frac{r_{0}^{2}}{\hat{R}^{2}}+\frac{a}{r_{0}}, \quad 0<r \leq r_{0} .
\end{gathered}
$$

For $a=0$,

$$
P_{0}(r)=r\left(r^{2}-\hat{R}^{2}\right), \quad J_{0}(r)=\int \frac{r d r}{\left|r^{2}-\hat{R}^{2}\right|^{3 / 2}} .
$$

Let us eliminate $\rho$ between (40) (with $a=0)$ and $\hat{R}^{2}=3 c^{2} /(8 \pi \kappa \rho)$ so that to obtain

$$
\frac{r_{0}}{\hat{R}}=\sqrt{\frac{2 \kappa M}{c^{2} r_{0}}} .
$$

The calculation of the dimensionless quantity (46) for $\alpha$-Centauri and Sun (at the present age

\begin{tabular}{|c|c|c|c|}
\hline Star & $r_{0}$ & $M$ & $r_{0} / \hat{R}$ \\
\hline$\alpha$-Centauri & $R_{\alpha}$ & $M_{\alpha}$ & 0.001896996991 \\
\hline $\begin{array}{cc} & \text { age } \\
\text { Sun } & t_{p} \\
& t_{p}+\Delta t\end{array}$ & $\begin{array}{c}R_{\odot} \\
R_{\odot}\left(t_{p}+\Delta t\right)\end{array}$ & $\begin{array}{c}M_{\odot} \\
M_{\odot}\left(t_{p}+\Delta t\right)\end{array}$ & $\begin{array}{c}0.002059672106 \\
0.00202464319\end{array}$ \\
\hline
\end{tabular}
$t_{p}=4.5 \times 10^{9}$ years, respectively, at age $t_{p}+\Delta t$ where $\Delta t=0.5 \mathrm{Gyr}$ ) is reported in Table 1 (gravitational constant $\kappa=6.67 \times 10^{-11} \mathrm{~kg}^{-1} \cdot \mathrm{m}^{3} \cdot \mathrm{s}^{-2}$ and speed of light in vacuum $c=$ $\left.299,792,458 \mathrm{~m} \cdot \mathrm{s}^{-1}\right)$. Let $M_{\odot}(t)$ and $R_{\odot}(t)$ be, respectively, Sun's mass and radius at age $t$, so that $M_{\odot}\left(t_{p}\right)=M_{\odot}=1.9884 \times 10^{30} \mathrm{~kg}$ and $R_{\odot}\left(t_{p}\right)=R_{\odot}=6.957 \times 10^{5} \mathrm{~km}$.

Table 1. Calculation of $r_{0} / \hat{R}$ for Sun and $\alpha$-Centauri.

Data used for $\alpha$-Centauri are $R_{\alpha}=0.145 R_{\odot}$ and $M_{\alpha}=0.123 M_{\odot}$ (cf. e.g., [7]); $R_{\odot}\left(t_{p}+\Delta t\right) / R_{\odot}=$ 1.03712 after $\Delta t$ Gyr from present age. Dimensionless quantity $r_{0} / \hat{R}$ decreases at later ages both as a function of $r_{0}$ (cf. Figure 1) and as a function of $M$ (as Sun expels $2.5 \times 10^{-14} M_{\odot}$ per year from emission of electromagnetic energy and ejection of matter with solar wind, cf. [8]). 


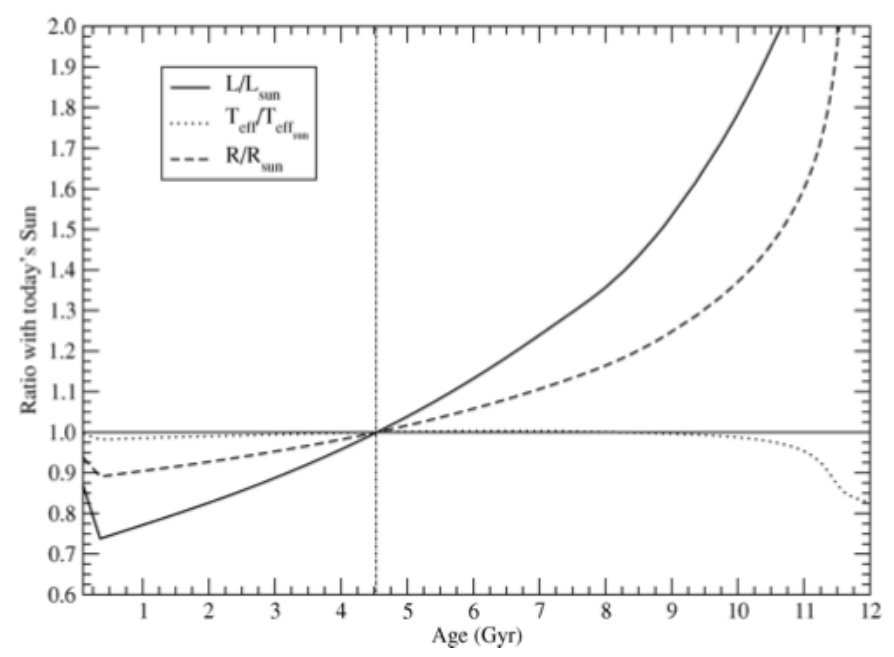

Figure 1. Evolution of the radius (effective temperature, and luminosity) of the Sun from the zero-age main sequence to the start of its red giant phase (cf. [9]).

This is practical evidence that $r_{0} / \hat{R}<<1$. In particular, $r / \hat{R} \leq r_{0} / \hat{R}<1$, so that sign $\left[P_{0}(r)\right]=-1$; hence,

$$
\begin{gathered}
J_{0}(r)=\int \frac{r d r}{\left(\hat{R}^{2}-r^{2}\right)^{3 / 2}}=\frac{1}{\sqrt{\hat{R}^{2}-r^{2}}}=\frac{1}{\hat{R}}\left(1-\frac{r^{2}}{\hat{R}^{2}}\right)^{-1 / 2}, \\
\sqrt{\frac{\left|P_{0}(r)\right|}{r}}=\hat{R}\left(1-\frac{r^{2}}{\hat{R}^{2}}\right)^{1 / 2}, \quad \epsilon_{0}=-1,
\end{gathered}
$$

and (43)-(74) become (for $a=0$ )

$$
A=\frac{3}{2}\left(1-\frac{r_{0}^{2}}{\hat{R}^{2}}\right)^{1 / 2}, \quad B \hat{R}^{2}=\frac{1}{2}
$$

(agreeing with (9.154) in [10] (p. 290)). Moreover,

$$
F_{0}(r)=3 \operatorname{sign}\left[P_{0}(r)\right] J_{0}(r)=-\frac{3}{\hat{R}}\left(1-\frac{r^{2}}{\hat{R}^{2}}\right)^{-1 / 2}
$$

hence,

$$
g_{00}=\frac{1}{4}\left(1-\frac{2 m}{r_{0}}\right) \frac{\left|P_{0}(r)\right|}{r}\left[F_{0}(r)-F_{0}\left(r_{0}\right)-2 \sqrt{\frac{r_{0}}{\left|P_{0}\left(r_{0}\right)\right|}}\right]^{2}=\left[\frac{1}{2} \sqrt{1-\frac{r^{2}}{\hat{R}^{2}}}-\frac{3}{2} \sqrt{1-\frac{r_{0}^{2}}{\hat{R}^{2}}}\right]^{2}
$$

(agreeing with (9.156) in [10] (p. 290)).

One has (with $a$ satisfying (38))

$$
-g_{11}=\left[1-\frac{r^{2}}{\hat{R}^{2}}+\frac{r_{0}}{r}\left(\frac{r_{0}^{2}}{\hat{R}^{2}}-\frac{2 m}{r_{0}}\right)\right]^{-1}, \quad P_{a}(r)=r^{3}-r_{0}^{3}-\hat{R}^{2}(r-2 m),
$$

hence

$$
g_{11}=\frac{\hat{R}^{2} r}{P_{a}(r)} .
$$


In particular, $g_{a}$ is only defined in (a region contained in) $P_{a}(r)<0$ (and $P_{a}\left(r_{0}\right)=-\hat{R}^{2}\left(r_{0}-2 m\right)<0$ because of $g_{00}>0$ for any $r \geq r_{0}$ for the exterior Schwarzschild solution), so that

$$
\begin{gathered}
F_{a}(r)-F_{a}\left(r_{0}\right)-2 \sqrt{\frac{r_{0}}{\left|P_{a}\left(r_{0}\right)\right|}}=3\left\{J_{a}\left(r_{0}\right)-J_{a}(r)\right\}-\frac{2}{\hat{R}}\left(1-\frac{2 m}{r_{0}}\right)^{-1 / 2}, \\
J_{a}\left(r_{0}\right)-J_{a}(r)=\int_{r}^{r_{0}} \frac{s^{5 / 2} d s}{\left(r_{0}^{3}-s^{3}+\hat{R}^{2} s-2 m \hat{R}^{2}\right)^{3 / 2}},
\end{gathered}
$$

hence (the $g_{00}$ coefficient of $g_{a}$ ),

$$
g_{00}=\frac{1}{4}\left(\frac{2 m}{r_{0}}-1\right) \frac{P_{a}(r)}{r}\left[\frac{2}{\hat{R}}\left(1-\frac{2 m}{r_{0}}\right)^{-1 / 2}+\int_{r_{0}}^{r} \frac{3 s^{5 / 2} d s}{\left(r_{0}^{3}-2 m \hat{R}^{2}+\hat{R}^{2} s-s^{3}\right)^{3 / 2}}\right]^{2},
$$

where $\hat{R}^{2}=r_{0}^{3} /(a+2 m)$. The hyperelliptic integral in (49) may be derived from the integral in (60), thought of as a parametric integral

$$
\frac{\partial}{\partial m} \int \frac{r^{5 / 2} d r}{\sqrt{r_{0}^{3}-r^{3}+\hat{R}^{2}(r-2 m)}}=\hat{R}^{2} \int \frac{r^{5 / 2} d r}{\left(r_{0}^{3}-2 m \hat{R}^{2}+\hat{R}^{2} r-r^{3}\right)^{3 / 2}}
$$

while the calculation of the antiderivative in (60) is addressed in Appendix A.

\section{Pressure Field}

To fully solve the boundary value problem (13)-(16), one is left with the determination of pressure field $p(r)$. To this end, we recall (24), implying (by $p(r) \geq 0$ and $\rho>0$ ) that $D>0$. Yet $D=\left(2 / \hat{R}^{2}\right) A$ or (by (43))

$$
D=-\frac{3 \epsilon_{a}}{\hat{R}^{2}}\left(1-\frac{2 m}{r_{0}}\right)^{1 / 2}
$$

yielding $\epsilon_{a}=-1$. Note that (by (6) and (39)) $P_{a}\left(r_{0}\right)=\frac{2 m-r_{0}}{a+2 m} r_{0}^{3}<0$ hence,

$$
-1=\epsilon_{a}=\operatorname{sign}\left\{2-3 \sqrt{-\frac{P_{a}\left(r_{0}\right)}{r_{0}}}\right\}
$$

or

$$
a+2 m<\frac{9 r_{0}^{2}}{4}\left(r_{0}-2 m\right)
$$

As a consequence of (40), $\rho=\rho_{a}$ where

$$
\rho_{a}=\frac{3}{4 \pi r_{0}^{3}}\left(M+\frac{c^{2}}{2 \kappa} a\right) .
$$

Let us substitute from (52), (50) (with $\epsilon_{a}=-1$ ) and (59) into (24). We obtain $p=p_{a}$ where

$$
\frac{p_{a}}{c^{2}}+\rho_{0}+\frac{3 c^{2}}{8 \kappa \pi r_{0}^{3}} a=-\frac{2}{C \hat{R}^{2}} \frac{\sqrt{\frac{r}{\left|P_{a}(r)\right|}}}{\left|v(a)-I_{a}(r)\right|}
$$


where we set

$$
\begin{gathered}
I_{a}(r)=\int_{r}^{r_{0}} \frac{s^{5 / 2} d s}{\left[r_{0}^{3}-s^{3}+\hat{R}^{2}(s-2 m)\right]^{3 / 2}}, \quad 0 \leq r \leq r_{0} . \\
v(a)=\frac{2}{3 \hat{R}}\left(1-\frac{2 m}{r_{0}}\right)^{-1 / 2}, \quad a>-2 m .
\end{gathered}
$$

For $a=0$, Formula (53) becomes

$$
\frac{p_{0}}{c^{2}}+\rho_{0}=2 \rho_{0}\left|3-\frac{1}{r_{0}}\left(\frac{r_{0}}{2 m}-1\right)^{-1 / 2}\left(\frac{r_{0}^{3}}{2 m}-r^{2}\right)^{1 / 2}\right|^{-1}
$$

(expressing the pressure field in the case considered by K. Schwarzschild, cf. op. cit.) (see Figure 2). We prove the following theorem: the following statements are equivalent: (i) $r_{0}>9 \mathrm{~m} / 4$ and (ii) there is an open neighbourhood of the origin $U_{0} \subset(-2 m,+\infty)$, such that, for any $a \in U_{0}$, pressure field $p_{a}(r)$ is a bounded function in the interval $0 \leq r \leq r_{0}$. With notations (54) and (55), pressure $p_{a}$ is bounded in $\left[0, r_{0}\right]$ if and only if $I_{a}(r) \neq v(a)$ for any $0 \leq r \leq r_{0}$. We first attack the implication (ii) $\Longrightarrow$ (i). In particular (under Hypothesis (ii)), $p_{0}$ is bounded in $\left[0, r_{0}\right]$, yielding

$$
I_{0}(r)-v(0) \neq 0, \quad \forall 0 \leq r \leq r_{0} ，
$$

where $v(0)=\frac{2}{3}\left(\frac{r_{0}^{3}}{2 m}-r_{0}^{2}\right)^{-1 / 2}$ hence (56) is equivalent to

$$
r^{2}+\frac{4 r_{0}^{3}}{m}-9 r_{0}^{2} \neq 0, \quad \forall 0 \leq r \leq r_{0}
$$

so that it must be $9 r_{0}^{2}-4 r_{0}^{3} / m<0$ or

$$
r_{0}>\frac{9 m}{4}
$$

which is (i). This is precisely the requirement (on structural parameters $r_{0}$ and $M$ ) discovered by $\mathrm{K}$. Schwarzschild (cf. op. cit., or formula (9.163) in [10] (p. 292)) that ought to hold in order that pressure $p_{0}(r)$ may never become infinite inside the fluid.

To prove the implication (i) $\Longrightarrow$ (ii) is to show that the previous result persists under a small 1-parameter variation of $p_{0}$ about $a=0$. Let us assume that requirement (57) is satisfied. Then, (56) holds, so that (as $I_{0}(r)$ is strictly decreasing in $0 \leq r \leq r_{0}$ )one has $I_{0}(0)<v(0)$. Let us consider auxiliary function $\psi(a)=I_{a}(0)-v(a)$ defined in $a>-2 m$. By the continuity of $\psi$, property $\psi(0)<0$ persists in some open neighbourhood $0 \in U_{0} \subset(-2 m,+\infty)$ i.e., $\psi(a)<0$ or $I_{a}(0)<v(a)$ for any $a \in U_{0}$. Lastly, as $I_{a}$ is strictly decreasing in $\left[0, r_{0}\right]$, we may conclude that

$$
\forall a \in U_{0}, \quad \forall 0 \leq r \leq r_{0}: I_{a}(r)<v(a) .
$$

Q.e.d. As a corollary, we may show that the maximum domain $\Omega \subset \mathbb{R}^{4}$ of the "physical" solution $\left(g_{a}, \rho_{a}, p_{a}\right)$ (obeying to the requirements $\rho_{a}(r) \geq 0, p_{a}(r) \geq 0$, and $p_{a}(r)$ is bounded in $\left.0 \leq r \leq r_{0}\right)$ is $\Omega=\mathbb{R} \times B_{r_{0}}(0)$, for any $a \in U_{0}$. Here $B_{r}(\mathbf{x})$ denotes the closed ball of radius $r>0$ and center $\mathbf{x} \in \mathbb{R}^{3}$.

Indeed, as $g_{00}>0$ and $g_{11}<0$ in $\Omega$, the domain is determined by the requirements $r \neq 0$, $P_{a}(r)<0$ and $I_{a}(r) \neq v(a)$. To compute $\Omega$, note first that $P_{a}^{\prime}(r)=3 r^{2}-r_{0}^{3} /(a+2 m)$ and 


$$
\operatorname{Crit}\left(P_{a}\right) \cap\left[0, r_{0}\right]= \begin{cases}\varnothing & \text { if } a+2 m<\frac{r_{0}}{3}, \\ \sqrt{\frac{r_{0}^{3}}{3(a+2 m)}} & \text { if } a+2 m \geq \frac{r_{0}}{3} .\end{cases}
$$

Consequently, if i) $a \geq 0$ then $P_{a}(r)<0$ for any $r \in\left[0, r_{0}\right]$, while if ii) $-2 m<a<0$, there is a unique $r(a) \in\left(0, r_{0}\right)$ such that $P_{a}[r(a)]=0, P_{a}(r)>0$ for any $r \in[0, r(a))$, and $P_{a}(r)<0$ for any $r \in\left(r(a), r_{0}\right]$.

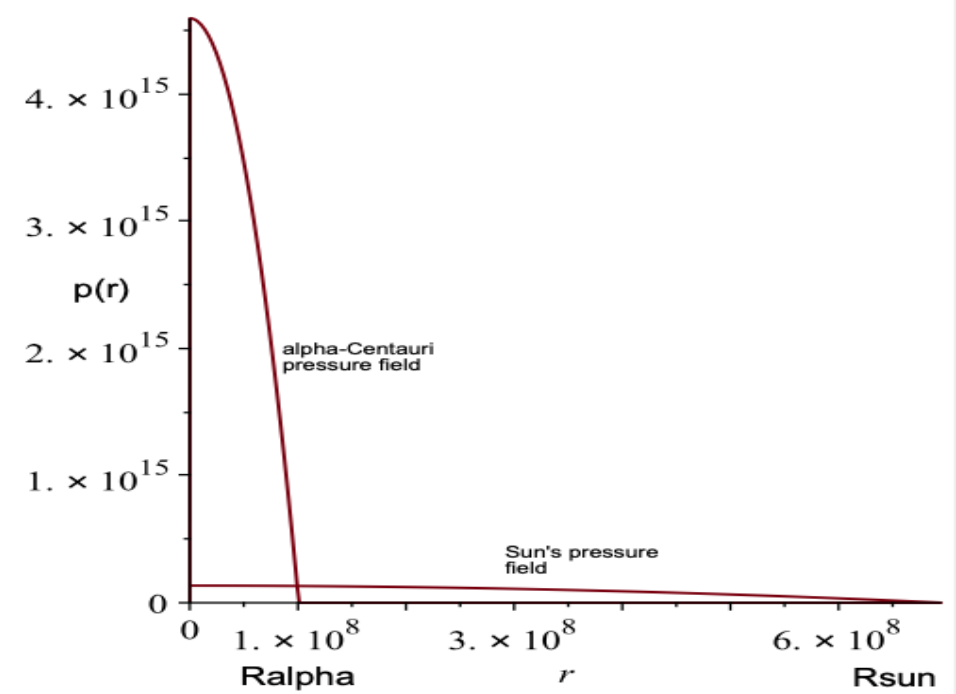

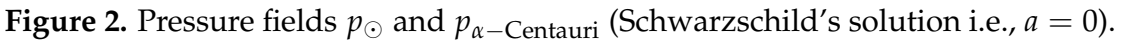

\section{Volume Calculations}

The "true" physical density is not constant as an effect of curvature i.e., the metric $g_{a}$ (with $a=r_{0}^{3} / \hat{R}^{2}-2 m$ ) varies in the fluid ambient, and the very notion of density depends on $g_{a}$. To compute the "physical" density, let us set $d s_{r}=\sqrt{-g_{11}} d r$ (the "physically meaningful" radial interval), i.e.,

$$
d s_{r}=\frac{\hat{R} r^{1 / 2}}{\sqrt{r_{0}^{3}-r^{3}+\hat{R}^{2}(r-2 m)}} d r
$$

Similarly, $d s_{\theta}=\sqrt{-g_{22}} d \theta=r d \theta$ and $d s_{\varphi}=\sqrt{-g_{33}} d \varphi=r \sin \theta d \varphi$. The "physical" volume element is then

$$
d V=d s_{r} d s_{\theta} d s_{\varphi}=\frac{\hat{R} r^{5 / 2} \sin \theta}{\sqrt{r_{0}^{3}-r^{3}+\hat{R}^{2}(r-2 m)}} d r d \theta d \varphi .
$$

Let us integrate in (59) over $0 \leq \theta \leq \pi, 0 \leq \varphi \leq 2 \pi$ and $0 \leq r \leq r_{0}$ so that to determine the total volume

$$
V=\int_{0}^{\pi} d \theta \int_{0}^{2 \pi} d \varphi \int_{0}^{r_{0}} \frac{\hat{R} r^{5 / 2} \sin \theta d r}{\sqrt{r_{0}^{3}-r^{3}+\hat{R}^{2}(r-2 m)}}=4 \pi \hat{R} \int_{0}^{r_{0}} \frac{r^{5 / 2} d r}{\sqrt{r_{0}^{3}-r^{3}+\hat{R}^{2}(r-2 m)}} .
$$


To compute $V$, we rely on considerations in Appendix A (reducing the underlying indefinite integral to the canonical Legendre form). Let us assume that

$$
a>\frac{2}{3 \sqrt{3}} \hat{R}, \quad \sigma>0, \quad \tau<\sigma^{2}<4 \tau,
$$

(yielding $\mathbf{m}=-h^{2}<0, \mathbf{m}^{\prime}=h^{\prime 2}>0$ and $\mathcal{A}<0$, with the notations in Appendix A). The first of the assumptions (61) yields $a>0$, and (61) is then equivalent to $a>a_{0}$, where $a_{0} \in \mathbb{R}$ is the only positive root of the polynomial $F(X)=X^{3}+2 m X^{2}-\frac{4 r_{0}^{3}}{27} \in \mathbb{R}[X]$. Indeed (by the boundary condition (38)) $a^{2}>4 \hat{R}^{2} / 27$ is equivalent (for positive $a$ ) to $F(a)>0$. Yet, $F(0)<0, F$ is monotonously increasing in $(0,+\infty)$, and $\lim _{X \rightarrow+\infty} F(X)=+\infty$ hence there is a unique $a_{0}>0$, such that $F\left(a_{0}\right)=0$. Lastly, the last two requirements in (61) are satisfied for every $a>a_{0}$. Next, note that

$$
\mu<r_{0}
$$

Indeed, starting from $\mu=\frac{1}{2 \sigma}\left[-\tau+\sqrt{\tau\left(\tau+2 \sigma^{2}\right)}\right]$ inequality (62) is equivalent to $\tau \sigma<2 r_{0}\left(\tau+r_{0} \sigma\right)$ or (by replacing $\tau$ from $\left.\sigma \tau=r_{0}^{3}-2 m \hat{R}^{2}\right) Q(\sigma)>0$ where $Q(x)=\frac{2 r_{0}^{2}}{r_{0}^{3}-2 m \hat{R}^{2}} x^{2}-x+2 r_{0}$. Yet $Q(x)>0$ for any $x \in \mathbb{R}$. Q.e.d.

Let us set

$$
g(x)=\frac{x^{5 / 2}}{\sqrt{r_{0}^{3}-x^{3}+\hat{R}^{2}(x-2 m)}}, \quad x^{3}-\hat{R}^{2} x+2 m \hat{R}^{2}-r_{0}^{3}<0 .
$$

The domain of $g$ is $(-\infty, \sigma)$ (cf. Table A1).Note that

$$
r_{0}<\sigma
$$

(hence, integral in (60) is well-defined).To prove (63) one observes first that

$$
r_{0}<\sigma \Longleftrightarrow 2 m<\sigma \Longrightarrow r_{0}^{2}-\hat{R}^{2}<\tau
$$

The remainder of the argument is by contradiction. If $r_{0} \geq \sigma$, then (by (64)) $\sigma \tau \geq 2 m\left(r_{0}^{2}-\hat{R}^{2}\right)$ or [again by $\sigma \tau=r_{0}^{3}-2 m \hat{R}^{2}$ ] $2 m \geq r_{0}$ [in contradiction with $\left(g_{S}\right)_{00}>0$ ]. As a corollary to (63)

$$
\frac{1}{h^{2}}=\left(\frac{v}{\mu}\right)^{2}<t_{o}^{2}
$$

where $t_{0}=\left(v-r_{0}\right) /\left(r_{0}-\mu\right)$. Indeed

$$
\left(\frac{v}{\mu}\right)^{2}<\left(\frac{v-r_{0}}{r_{0}-\mu}\right)^{2} \Longleftrightarrow r_{0}\left(v^{2}-\mu^{2}\right)+2 \mu \nu(\mu-v)<0 \Longleftrightarrow(63)
$$

[by substitution from $\mu v=-\frac{\tau}{2}, \mu-v=\frac{1}{\sigma} \sqrt{\tau\left(\tau+2 \sigma^{2}\right)}$ and $\left.v^{2}-\mu^{2}=\frac{\tau}{\sigma^{2}} \sqrt{\tau\left(\tau+2 \sigma^{2}\right)}\right]$. As part of the reduction to the canonical form scheme one needs to apply a homographic change of variables $x=(\mu t+v)(t+1)^{-1}$ under the integral sign. Yet the pre-images $\left\{-v / \mu, t_{0}\right\}$ of $\left\{0, r_{0}\right\}$ are separated by the singularity $t=-1$; hence, one needs to split (by taking into account (62))integral $\int_{0}^{r_{0}} g(x) d x$ as $\int_{0}^{\mu} g(x) d x+\int_{\mu}^{r_{0}} g(x) d x$ and apply the transformations $x:(-1,+\infty) \rightarrow(-\infty, \mu)$ and 
$x:(-\infty,-1) \rightarrow(\mu,+\infty)$, respectively. This leads to generalized integrals, so a bit of extra pedantry is needed to correctly apply change of variable theorems, i.e.,

$$
\int_{0}^{r_{0}} g(x) d x=\lim _{\substack{\alpha \rightarrow 0^{+} \\ \beta \rightarrow \mu^{-}}} \int_{\alpha}^{\beta} g(x) d x+\lim _{\substack{\alpha \rightarrow \mu^{+} \\ \beta \rightarrow r_{0}^{-}}} \int_{\alpha}^{\beta} g(x) d x=\lim _{\substack{\alpha \rightarrow 0^{+} \\ \beta \rightarrow \mu^{-}}} I_{\alpha \beta}+\lim _{\substack{\alpha \rightarrow \mu^{+} \\ \beta \rightarrow r_{0}^{-}}} I_{\alpha \beta}
$$

where

$$
I_{\alpha \beta}:=\int_{t_{\alpha}}^{t_{\beta}} \frac{\mathcal{R}^{*}(t) d t}{\sqrt{\mathcal{A}\left(1-h^{2} t^{2}\right)\left(1+h^{\prime 2} t^{2}\right)}}
$$

with A) $1<-v / \mu<t_{\alpha}<t_{\beta}$, respectively, B) $t_{\alpha}<t_{\beta}<t_{r_{0}}<-1$. Here, $t_{\gamma}:=(v-\gamma)(\gamma-\mu)^{-1}$ [so that $t_{0}=t_{r_{0}}$. Next, one decomposes $\mathcal{R}^{*}$ into even and odd components, i.e., $\mathcal{R}^{*}(t)=\mathcal{R}_{1}\left(t^{2}\right)+t \mathcal{R}_{2}\left(t^{2}\right)$; hence, (in Case (A))

$$
\lim _{\substack{\alpha \rightarrow 0^{+} \\ \beta \rightarrow \mu^{-}}} I_{\alpha \beta}=\lim _{\substack{\alpha \rightarrow 0^{+} \\ \beta \rightarrow \mu^{-}}} \int_{t_{\alpha}}^{t_{\beta}} \frac{\left[\mathcal{R}_{1}\left(t^{2}\right)+t \mathcal{R}_{2}\left(t^{2}\right)\right] d t}{\sqrt{\mathcal{A}\left(1-h^{2} t^{2}\right)\left(1+h^{\prime 2} t^{2}\right)}}=
$$

(by $h t=\left(1-z^{2}\right)^{-1 / 2}$ with $0<z<1$ )

$$
=\lim _{\substack{\alpha \rightarrow 0^{+} \\ \beta \rightarrow \mu^{-}}}\left\{\frac{k(\mu+v)}{h \sqrt{-\mathcal{A}}} \int_{z_{\alpha}}^{z_{\beta}} \frac{\mathcal{R}_{1}^{*}\left(z^{2}\right) d z}{\sqrt{\left(1-z^{2}\right)\left(1-k^{2} z^{2}\right)}}+\left.\mathcal{S}\left[\frac{1}{h^{2}\left(1-z^{2}\right)}\right]\right|_{z_{\alpha}} ^{z_{\beta}}\right\}=
$$

(where $z_{\alpha}=\sqrt{1-1 /\left(h^{2} t_{\alpha}^{2}\right)}$ or $[$ by $z=\sin \phi, 0<\phi<\pi / 2$ )

$$
\begin{gathered}
=\lim _{\substack{\alpha \rightarrow 0^{+} \\
\beta \rightarrow \mu^{-}}}\left\{\frac { k ( \mu + v ) v ^ { 2 } } { h \mathcal { M } ^ { \prime } \sqrt { - \mathcal { A } } } \left[\left.8 k^{2} \cot \phi \sqrt{1-k^{2} \sin ^{2} \phi}\right|_{\phi_{\alpha}} ^{\phi_{\beta}}\right.\right. \\
+\left(1-8 k^{2}\right) \int_{\phi_{\alpha}}^{\phi_{\beta}} \frac{d \phi}{\sqrt{1-k^{2} \sin ^{2} \phi}+8 k^{2} \int_{\phi_{\alpha}}^{\phi_{\beta}} \sqrt{1-k^{2} \sin ^{2} \phi} d \phi} \\
\left.\left.+\left[8 k^{2}\left(1-k^{2}\right)-1\right] \int_{\phi_{\alpha}}^{\phi_{\beta}} \frac{d \phi}{\left(1-k^{2} \sin ^{2} \phi\right) \sqrt{1-k^{2} \sin ^{2} \phi}}\right]+\left.\mathcal{S}\left(\frac{1}{h^{2} \cos ^{2} \phi}\right)\right|_{\phi_{\alpha}} ^{\phi_{\beta}}\right\}
\end{gathered}
$$

(where $\phi_{\alpha}=\arcsin z_{\alpha}$ ) and

$$
\left.\lim _{\substack{\alpha \rightarrow 0^{+} \\ \beta \rightarrow \mu^{-}}}\left[\frac{8 k^{3}(\mu+v) v^{2}}{h \mathcal{M}^{\prime} \sqrt{-\mathcal{A}}} \cot \phi \sqrt{1-k^{2} \sin ^{2} \phi}+\mathcal{S}\left(\frac{1}{h^{2} \cos ^{2} \phi}\right)\right]\right|_{\phi_{\alpha}} ^{\phi_{\beta}}
$$

(by (A22))

$$
=\left.\frac{4 k^{3}(\mu+v)^{2} \mu}{h^{2}(v-\mu)(2 \mu+v) \sqrt{-\mathcal{A}}} \lim _{\substack{\alpha \rightarrow 0^{+} \\ \beta \rightarrow \mu^{-}}} F(\phi)\right|_{\phi_{\alpha}} ^{\phi_{\beta}}=\frac{4 k^{3}\left(3-4 k^{2}\right)}{h^{2} \sqrt{1-k^{2}}} \frac{(\mu+v)^{2} \mu}{(v-\mu)(2 \mu+v) \sqrt{-\mathcal{A}}}
$$


where

$$
F(\phi):=\frac{1}{\sin \phi}\left[\frac{2+\left(1-4 k^{2}\right) \sin ^{2} \phi}{\sqrt{1-k^{2} \sin ^{2} \phi}}-2 \cos \phi \sqrt{1-k^{2} \sin ^{2} \phi}\right]
$$

Indeed, $\alpha \rightarrow 0^{+}, \beta \rightarrow \mu^{-} \Longrightarrow \phi_{\alpha} \rightarrow 0^{+}, \phi_{\beta} \rightarrow\left(\frac{\pi}{2}\right)^{-}$and

$$
\lim _{\phi \rightarrow 0} F(\phi)=0, \quad \lim _{\phi \rightarrow \frac{\pi}{2}} F(\phi)=\frac{3-4 k^{2}}{\sqrt{1-k^{2}}}
$$

Lastly, (in Case (A))

$$
\begin{gathered}
\lim _{\substack{\alpha \rightarrow 0^{+} \\
\beta \rightarrow \mu^{-}}} I_{\alpha \beta}=\frac{4 k^{3}\left(3-4 k^{2}\right)}{h^{2} \sqrt{1-k^{2}}} \frac{(\mu+v)^{2} \mu}{(v-\mu)(2 \mu+v) \sqrt{-\mathcal{A}}} \\
+\frac{k v(\mu+v)^{2}}{h(v-\mu)(2 \mu+v) \sqrt{-\mathcal{A}}}\left[\left(1-8 k^{2}\right) \int_{0}^{\pi / 2} \frac{d \phi}{\sqrt{1-k^{2} \sin ^{2} \phi}+8 k^{2} \int_{0}^{\pi / 2} \sqrt{1-k^{2} \sin ^{2} \phi} d \phi}\right. \\
\left.+\left[8 k^{2}\left(1-k^{2}\right)-1\right] \int_{0}^{\pi / 2} \frac{d \phi}{\left(1-k^{2} \sin ^{2} \phi\right) \sqrt{1-k^{2} \sin ^{2} \phi}}\right]
\end{gathered}
$$

or

$$
\begin{gathered}
\lim _{\alpha \rightarrow 0^{+}} I_{\alpha \beta}=\frac{k v(\mu+v)^{2}}{h(v-\mu)(2 \mu+v) \sqrt{-\mathcal{A}}} \times \\
\beta \rightarrow \mu^{-} \\
\times\left\{\left(1-8 k^{2}\right) \mathbf{F}\left(k, \frac{\pi}{2}\right)+8 k^{2} \mathbf{E}\left(k, \frac{\pi}{2}\right)+\left[8 k^{2}\left(1-k^{2}\right)-1\right] \Pi\left(k^{2}, k\right)+\frac{4 k^{2}\left(4 k^{2}-3\right)}{\sqrt{1-k^{2}}}\right\}
\end{gathered}
$$

with the Legendre notations

$$
\begin{gathered}
\mathbf{F}(k, \theta)=\int_{0}^{\theta} \frac{d \phi}{\sqrt{1-k^{2} \sin ^{2} \phi}}, \quad \mathbf{E}(k, \theta)=\int_{0}^{\theta} \sqrt{1-k^{2} \sin ^{2} \phi} d \phi, \\
\Pi(\ell, k)=\int_{0}^{\pi / 2} \frac{d \phi}{\left(1-\ell \sin ^{2} \phi\right) \sqrt{1-k^{2} \sin ^{2} \phi}},
\end{gathered}
$$

for the abelian integrals of the first, second, and third kind of module $k$. As to Case (B),

$$
\lim _{\substack{\alpha \rightarrow \mu^{+} \\ \beta \rightarrow r_{0}^{-}}} I_{\alpha \beta}=\lim _{\substack{\alpha \rightarrow \mu^{+} \\ \beta \rightarrow r_{0}^{-}}} \int_{t_{\alpha}}^{t_{\beta}} \frac{\mathcal{R}^{*}(t) d t}{\sqrt{\mathcal{A}\left(1-h^{2} t^{2}\right)\left(1+h^{\prime 2} t^{2}\right)}}=
$$

$[$ by setting $s=-t]$

$$
=\lim _{\substack{\alpha \rightarrow \mu^{+} \\ \beta \rightarrow r_{0}^{-}}}\left[\int_{-t_{\beta}}^{-t_{\alpha}} \frac{\mathcal{R}_{1}\left(t^{2}\right) d t}{\sqrt{\mathcal{A}\left(1-h^{2} t^{2}\right)\left(1+h^{\prime 2} t^{2}\right)}}-\int_{-t_{\beta}}^{-t_{\alpha}} \frac{t \mathcal{R}_{2}\left(t^{2}\right) d t}{\sqrt{\mathcal{A}\left(1-h^{2} t^{2}\right)\left(1+h^{\prime 2} t^{2}\right)}}\right]=
$$




$$
\begin{aligned}
& {\left[\text { with }-t_{\alpha}>-t_{\beta}>-t_{r_{0}}>\frac{1}{h}\right] \text { or }\left[\text { by } h t=\left(1-z^{2}\right)^{-1 / 2}, 0<z<1\right]} \\
& =\lim _{\substack{\alpha \rightarrow \mu^{+} \\
\beta \rightarrow r_{0}^{-}}}\left[-\frac{(\mu+v) k}{h \sqrt{-\mathcal{A}}} \int_{z_{\alpha}}^{z_{\beta}} \frac{\mathcal{R}_{1}^{*}\left(z^{2}\right) d z}{\sqrt{\left(1-z^{2}\right)\left(1-k^{2} z^{2}\right)}}+\left.\mathcal{S}\left(\frac{1}{h^{2}\left(1-z^{2}\right)}\right)\right|_{z_{\alpha}} ^{z_{\beta}}\right] \\
& =\lim _{\alpha \rightarrow \mu^{+}}\left\{-\frac{k(\mu+v) v^{2}}{h \mathcal{M}^{\prime} \sqrt{-\mathcal{A}}}\left[\left.8 k^{2} \cot \phi \sqrt{1-k^{2} \sin ^{2} \phi}\right|_{\phi_{\alpha}} ^{\phi_{\beta}}\right.\right. \\
& +\left(1-8 k^{2}\right) \int_{\phi_{\alpha}}^{\phi_{\beta}} \frac{d \phi}{\sqrt{1-k^{2} \sin ^{2} \phi}+8 k^{2} \int_{\phi_{\alpha}}^{\phi_{\beta}} \sqrt{1-k^{2} \sin ^{2} \phi} d \phi} \\
& \left.\left.+\left[8 k^{2}\left(1-k^{2}\right)-1\right] \int_{\phi_{\alpha}}^{\phi_{\beta}} \frac{d \phi}{\left(1-k^{2} \sin ^{2} \phi\right) \sqrt{1-k^{2} \sin ^{2} \phi}}\right]+\left.\mathcal{S}\left(\frac{1}{h^{2} \cos ^{2} \phi}\right)\right|_{\phi_{\alpha}} ^{\phi_{\beta}}\right\} .
\end{aligned}
$$

Note that $\alpha \rightarrow \mu^{+} \Longrightarrow \phi_{\alpha}=\arcsin \sqrt{1-\frac{1}{h^{2}}\left(\frac{\alpha-\mu}{v-\alpha}\right)^{2}} \rightarrow \frac{\pi}{2}$ and $\beta \quad \rightarrow \quad r_{0}^{-} \quad \Longrightarrow$ $\phi_{\beta}=\arcsin \sqrt{1-\frac{1}{h^{2}}\left(\frac{\beta-\mu}{v-\beta}\right)^{2}} \rightarrow \arcsin \sqrt{1-\frac{1}{h^{2} t_{o}^{2}}}=: \phi_{0}$. Summing up our previous calculations

$$
\mathcal{S}\left(\frac{1}{h^{2} \cos ^{2} \phi}\right)=\frac{4 k^{3} \mu(\mu+v)^{2}}{h^{2}(v-\mu)(2 \mu+v) \sqrt{-\mathcal{A}}} \frac{2+\left(1-4 k^{2}\right) \sin ^{2} \phi}{\sin \phi \sqrt{1-k^{2} \sin ^{2} \phi}} ;
$$

hence,

$$
\begin{gathered}
\left.\lim _{\substack{\alpha \rightarrow \mu^{+} \\
\beta \rightarrow r_{0}^{-}}}\left\{-\frac{8 k^{3}(\mu+v) v^{2}}{h \mathcal{M}^{\prime} \sqrt{-\mathcal{A}}} \cot \phi \sqrt{1-k^{2} \sin ^{2} \phi}+\mathcal{S}\left(\frac{1}{h^{2} \cos ^{2} \phi}\right)\right\}\right|_{\phi_{\alpha}} ^{\phi_{\beta}} \\
=\frac{4 k^{3} \mu(\mu+v)^{2}}{h^{2}(v-\mu)(2 \mu+v) \sqrt{-\mathcal{A}}}\left[G\left(\phi_{0}\right)+\frac{4 k^{2}-3}{\sqrt{1-k^{2}}}\right]
\end{gathered}
$$

where

$$
G(\phi):=\frac{1}{\sin \phi}\left[\frac{2+\left(1-4 k^{2}\right) \sin ^{2} \phi}{\sqrt{1-k^{2} \sin ^{2} \phi}}+2 \cos \phi \sqrt{1-k^{2} \sin ^{2} \phi}\right] .
$$

Thus,

$$
\begin{gathered}
\lim _{\substack{\alpha \rightarrow \mu^{+} \\
\beta \rightarrow r_{0}^{-}}} I_{\alpha \beta}=\frac{k(\mu+v)^{2} v}{h(v-\mu)(2 \mu+v) \sqrt{-\mathcal{A}}} \times \\
\times\left[\left(1-8 k^{2}\right) \int_{\phi_{o}}^{\pi / 2} \frac{d \phi}{\sqrt{1-k^{2} \sin ^{2} \phi}}+8 k^{2} \int_{\phi_{o}}^{\pi / 2} \sqrt{1-k^{2} \sin ^{2} \phi} d \phi\right. \\
\left.+\left[8 k^{2}\left(1-k^{2}\right)-1\right] \int_{\phi_{o}}^{\pi / 2} \frac{d \phi}{\left(1-k^{2} \sin ^{2} \phi\right) \sqrt{1-k^{2} \sin ^{2} \phi}}\right]
\end{gathered}
$$




$$
+\frac{4 k^{3} \mu(\mu+v)^{2}}{h^{2}(v-\mu)(2 \mu+v) \sqrt{-\mathcal{A}}}\left[G\left(\phi_{0}\right)+\frac{4 k^{2}-3}{\sqrt{1-k^{2}}}\right]
$$

and

$$
G\left(\phi_{0}\right)=\frac{1}{\sqrt{h^{2} t_{o}^{2}-1}}\left[\frac{\left(3-4 k^{2}\right) h^{2} t_{o}^{2}+4 k^{2}-1}{\sqrt{k^{2}+\left(1-k^{2}\right) h^{2} t_{o}^{2}}}-\frac{2}{h t_{o}} \sqrt{k^{2}+\left(1-k^{2}\right) h^{2} t_{o}^{2}}\right] .
$$

Then (in Case (B))

$$
\begin{gathered}
\lim _{\substack{\alpha \rightarrow \mu^{+} \\
\beta \rightarrow r_{0}^{-}}} I_{\alpha \beta}=\frac{k(\mu+v)^{2} v}{h(v-\mu)(2 \mu+v) \sqrt{-\mathcal{A}}} \times \\
\times\left\{\left(1-8 k^{2}\right)\left[\mathbf{F}\left(k, \frac{\pi}{2}\right)-\mathbf{F}\left(k, \phi_{0}\right)\right]+8 k^{2}\left[\mathbf{E}\left(k, \frac{\pi}{2}\right)-\mathbf{E}\left(k, \phi_{0}\right)\right]\right. \\
+\left[8 k^{2}\left(1-k^{2}\right)-1\right]\left[\Pi\left(k^{2}, k\right)-\int_{0}^{\phi_{0}} \frac{d \phi}{\left.\left(1-k^{2} \sin ^{2} \phi\right) \sqrt{1-k^{2} \sin ^{2} \phi}\right]}\right] \\
+\frac{4 k^{3} \mu(\mu+v)^{2}}{h^{2}(v-\mu)(2 \mu+v) \sqrt{-\mathcal{A}}}\left[G\left(\phi_{0}\right)+\frac{4 k^{2}-3}{\sqrt{1-k^{2}}}\right] .
\end{gathered}
$$

Summing up (by (66)-(68))

$$
\begin{gathered}
\int_{0}^{r_{0}} g(x) d x=\frac{k(\mu+v)^{2} v}{h(v-\mu)(2 \mu+v) \sqrt{-\mathcal{A}}} \times \\
\times\left\{\left(1-8 k^{2}\right)\left[2 \mathbf{F}\left(k, \frac{\pi}{2}\right)-\mathbf{F}\left(k, \phi_{0}\right)\right]+8 k^{2}\left[2 \mathbf{E}\left(k, \frac{\pi}{2}\right)-\mathbf{E}\left(k, \phi_{0}\right)\right]\right. \\
\left.+\left[8 k^{2}\left(1-k^{2}\right)-1\right]\left[2 \Pi\left(k^{2}, k\right)-\int_{0}^{\phi_{0}} \frac{d \phi}{\left(1-k^{2} \sin ^{2} \phi\right) \sqrt{1-k^{2} \sin ^{2} \phi}}\right]\right\} \\
+\frac{4 k^{3} \mu(\mu+v)^{2}}{h^{2}(v-\mu)(2 \mu+v) \sqrt{-\mathcal{A}}} \frac{1}{\sqrt{h^{2} t_{o}^{2}-1}}\left[\frac{\left(3-4 k^{2}\right) h^{2} t_{o}^{2}+4 k^{2}-1}{\sqrt{k^{2}+\left(1-k^{2}\right) h^{2} t_{o}^{2}}}-\frac{2}{h t_{o}} \sqrt{k^{2}+\left(1-k^{2}\right) h^{2} t_{o}^{2}}\right] .
\end{gathered}
$$

Going back to (60), volume $V(a)$ of region $0 \leq r \leq r_{0}$, whose geometry is governed by $g_{a}$, is

$$
V(a)=4 \pi \hat{R} I(a), \quad I(a):=\int_{0}^{r_{0}} \frac{r^{5 / 2} d r}{\sqrt{\frac{a+r}{a+2 m} r_{0}^{3}-r^{3}}} .
$$

In particular, for $a=0\left[\right.$ as (38) with $a=0$ yields $\left.\hat{R}^{2}=\frac{r_{0}^{3}}{2 m}\right]$

$$
V(0)=4 \pi \int_{0}^{r_{0}} \frac{r^{2} d r}{\sqrt{1-\left(\frac{r}{\hat{R}}\right)^{2}}}=2 \pi \hat{R}^{3} f\left(\frac{r_{0}}{\hat{R}}\right), \quad f(x)=\arcsin x-x \sqrt{1-x^{2}}, \quad|x| \leq 1 .
$$


This is the case examined by K. Schwarzschild (cf. op. cit.); practical evidence was previously gathered that $r_{0} / \hat{R}<<1$; hence, we may consider the truncated Taylor expansion of $V(0)$ as a function of $r_{0} / \hat{R}$, i.e., for every $n \in \mathbb{Z}_{+}$

$$
\begin{gathered}
V(0)=2 \pi \hat{R}^{3}\left[\sum_{k=0}^{n}(-1)^{k} a_{k}\left(\frac{r_{0}}{\hat{R}}\right)^{2 k+1}+R_{2 n+2}\left(\frac{r_{0}}{\hat{R}} ; 0\right)\right], \\
a_{k}=\frac{1}{2 k+1}\left(\begin{array}{c}
-1 / 2 \\
k
\end{array}\right)-\left(\begin{array}{c}
1 / 2 \\
k
\end{array}\right), 0 \leq k \leq n, \\
R_{2 n+2}(x ; 0)=(-1)^{n+1}\left\{\left(\begin{array}{c}
-1 / 2 \\
n+1
\end{array}\right) \int_{0}^{x} t^{2 n+2}\left[1-\eta(t)^{2}\right]^{-n-1 / 2}\right. \\
\left.-\left(\begin{array}{c}
1 / 2 \\
n+1
\end{array}\right) x^{2 n+3}\left[1-\eta(x)^{2}\right]^{-n-1 / 2}\right\}, \\
\left(\begin{array}{c}
\alpha \\
0
\end{array}\right)=1, \quad\left(\begin{array}{c}
\alpha \\
k
\end{array}\right)=\frac{\alpha(\alpha-1) \cdots(\alpha-k+1)}{k !}, \alpha \in \mathbb{R}, k \in \mathbb{N},
\end{gathered}
$$

for some function $\eta:[-1,1] \rightarrow \mathbb{R}$ such that $\eta(x)$ lies between 0 and $x$, or

$$
V(0)=\frac{4 \pi r_{0}^{3}}{3}\left[1+\frac{3}{10}\left(\frac{r_{0}}{\hat{R}}\right)^{2}+\frac{3}{2} \sum_{k=3}^{n}(-1)^{k} a_{k}\left(\frac{r_{0}}{\hat{R}}\right)^{2(k-1)}+\frac{3}{2}\left(\frac{r_{0}}{\hat{R}}\right)^{-3} R_{2 n+2}\left(\frac{r_{0}}{\hat{R}} ; 0\right)\right]
$$

and

$$
\left|\frac{3}{2}\left(\frac{r_{0}}{\hat{R}}\right)^{-3} R_{2 n+2}\left(\frac{r_{0}}{\hat{R}} ; 0\right)\right| \leq \frac{3\left(n+\frac{5}{4}\right) 2^{n+\frac{1}{2}}}{(n+1)(2 n+3)}\left(\frac{r_{0}}{\hat{R}}\right)^{2 n} .
$$

Estimate (71) follows from

$$
\left|R_{2 n+2}(x ; 0)\right| \leq \frac{n+\frac{5}{4}}{(n+1)(2 n+3)}\left(2 x^{2}\right)^{n+\frac{3}{2}}, \quad|x|<\frac{1}{\sqrt{2}} .
$$

Indeed, if for instance $x>0$, then $0<\eta<x$ yields $1 /\left(1-\eta^{2}\right)<1 / 2$; hence,

$$
\left|\int_{0}^{x} t^{2 n+2}\left[1-\eta(t)^{2}\right]^{-n-\frac{3}{2}} d t\right| \leq \frac{\left(2 x^{2}\right)^{n+\frac{3}{2}}}{2 n+3}
$$

and $\left(\begin{array}{c}-1 / 2 \\ n+1\end{array}\right)=\frac{(-1)^{n+1}}{2^{n+1}} \frac{(2 n+1) ! !}{(n+1) !}$, so that

$$
\left|\left(\begin{array}{c}
-1 / 2 \\
n+1
\end{array}\right)\right|=\frac{3 \times 5 \cdots \cdots(2 n+1)}{2^{n+1}(n+1) !} \leq \frac{4 \times 6 \cdots \cdots(2 n+2)}{2^{n+1}(n+1) !}=\frac{1}{2}
$$

i.e.,

$$
\left|\left(\begin{array}{c}
-1 / 2 \\
n+1
\end{array}\right)\right| \leq \frac{1}{2}
$$

Similarly,

$$
\left|x^{2 n+3}\left[1-\eta(x)^{2}\right]^{-n-\frac{1}{2}}\right| \leq \frac{1}{2}\left(2 x^{2}\right)^{n+\frac{3}{2}}
$$


and $\left(\begin{array}{c}1 / 2 \\ n+1\end{array}\right)=\frac{(-1)^{n}}{2^{n+1}} \frac{(2 n-1) ! !}{(n+1) !}$ so that

$$
\left|\left(\begin{array}{c}
1 / 2 \\
n+1
\end{array}\right)\right| \leq \frac{1}{2(n+1)} .
$$

Lastly, estimates (73)-(76) yield (72) for any $0<x<1 / \sqrt{2}$. Of course, the use of (74) and (76) does not lead to an optimal estimate on $\left|R_{2 n+2}(x ; 0)\right|((72)$ is to be preferred only for its mathematical elegance).

\section{Conclusions and Open Problems}

In the present paper, we examined a fundamental issue, present in most textbooks nowadays on general relativity and gravitation theory, i.e., the derivation of the interior Schwarzschild solution $g_{0}$ (cf. e.g., [10] (pp. 280-295)) and the resulting simple model of a star. To determine $g_{0}$ (and actually $\left(g_{0}, \rho_{0}, p_{0}\right)$ where $\rho_{0}$ is the (constant) density and $p_{0}$ the (radial) pressure field), one looks for a line element $d s$ of the form $d s^{2}=e^{v(r)} c^{2} d t^{2}-\left[e^{\lambda(r)} d r^{2}+r^{2}\left(d \theta^{2}+\sin ^{2} \theta d \varphi^{2}\right)\right]$ and needs to solve the boundary value problem (13)-(16) for $(\lambda, \mu, \rho, p)$. The general solution of the ODE system (13)-(15) contains three constants of integration $A, B, a \in \mathbb{R}$, and the given boundary conditions determine the first two, while the third is but subject to the constraint $a>-2 m$. This leads to a 1-parameter family of solutions $\left\{\left(\lambda_{a}, \mu_{a}, \rho_{a}, p_{a}\right)\right\}_{a>-2 m}$ to (13)-(16) with the property that $\lambda_{a}(r)$ is bounded at $r=0$ if and only if $a=0$. K. Schwarzschild discarded the solutions with $a \neq 0$ on the ground that the determinant of each $g_{a}$ with $a \neq 0$ vanishes at $r=0$; this is, of course, also the case for $g_{0}$, and the question arises whether any of the additional solutions $g_{a}$ is physically acceptable, thus leading to alternative geometric descriptions of the interior of a star of incompressible fluid. We explicitly determine the solutions $\left\{\left(g_{a}, \rho_{a}, p_{a}\right)\right\}_{a>-2 m}$ and show that the requirement (57) i.e., $r_{0}>9 m / 4$, which in Schwarzschild's work is equivalent to $p_{0}(r)$ staying finite in $r \leq r_{0}$, is also equivalent to the boundedness of $p_{a}(r)$ in $r \leq r_{0}$, though only for $a$ lying in some open neighbourhood $U_{0} \subset(-2 m,+\infty)$ of $a=0$. It should be observed that estimate (6) i.e., $r_{0}>2 m$ is slightly improved by estimate (57); (6) $\Longleftrightarrow M<\frac{c^{2} r_{0}}{2 \kappa}$ and (57) $\Longleftrightarrow M<\frac{4}{9} \frac{c^{2} r_{0}}{2 \kappa}$, so that the requirement (57) provides a slightly smaller upper bound on the amount $M$ of fluid that can be packed into a ball of radius $r_{0}$, in agreement with the geometry of space-time as described by the Lorentzian metric $g=\left\{\begin{array}{ll}g_{a} & r \leq r_{0} \\ g_{S} & r \geq r_{0}\end{array}\right.$. K. Schwarzschild used metric tensor $g_{0}$ to compute the volume of the region $r \leq r_{0}$

$$
V(0)=\int_{0}^{\pi} d \theta \int_{0}^{2 \pi} d \varphi \int_{0}^{r_{0}} \frac{r^{2} \sin \theta d r}{\sqrt{1-\frac{r^{2}}{\hat{R}^{2}}}}
$$

and derived the truncated Taylor development of $V(0)$ with respect to the parameter $r_{0} / \hat{R}$

$$
V(0)=\frac{4 \pi r_{0}^{3}}{3}\left[1+\frac{3}{10}\left(\frac{r_{0}}{\hat{R}}\right)^{2}+O\left(\frac{r_{0}}{\hat{R}}\right)^{4}\right] .
$$

When the geometry is described by $g_{0}$, the parameter $r_{0} / \hat{R}$ is $<<1$, as may be argued by invoking experimental evidence, and development (77) gives a fair approximation to volume $V(0)$. We slightly improved Schwarzschild's result by developing $V(0)$ to arbitrary fixed order (cf. our (70) in §3) and providing a mathematically elegant estimate on the Taylor rest (cf. (71) in §3)

$$
\left|R_{2 n+2}\left(\frac{r_{0}}{\hat{R}} ; 0\right)\right| \leq \frac{\left(n+\frac{5}{4}\right) 2^{n+\frac{3}{2}}}{(n+1)(2 n+3)}\left(\frac{r_{0}}{\hat{R}}\right)^{2 n+3} .
$$


On the basis of our discussion of the parameter $r_{0} / \hat{R}$ in $\S 1$ for the Sun, one has $r_{0} / \hat{R}<<1$ beyond Sun's present age, and Formulas (70) and (71) remain accurate up to the start of Sun's red giant phase. The calculation of the volume

$$
V(a)=\int_{0}^{\pi} d \theta \int_{0}^{2 \pi} d \varphi \int_{0}^{r_{0}} \frac{\hat{R} r^{5 / 2} \sin \theta d r}{\sqrt{r_{0}^{3}-r^{3}+\hat{R}^{2}(r-2 m)}}
$$

turned out to be more involved, as depending upon the evaluation of an abelian integral that may not be expressed in terms of algebraic functions, and which we can only reduce to the canonical Legendre form (cf. (60) and (69) in §3) confined to case $a>a_{0}$ for some constant $a_{0}>0$ (whose precise description is given in §3). Going back to (38), one has $\frac{r_{0}}{\hat{R}}=\sqrt{\frac{a+2 m}{r_{0}}}$ hence $\lim _{a \rightarrow+\infty}\left(r_{0} / \hat{R}\right)=+\infty$. Of course, one should take into account the upper bound on $a$ imposed by (51) implying

$$
\frac{r_{0}}{\hat{R}}<\frac{3 r_{0}}{2}\left(1-\frac{2 m}{r_{0}}\right)^{1 / 2}
$$

However, the right-hand side of (78) is in general $>1$ (hence truncated Taylor approximation of $V(a)$ as a function of $r_{0} / \hat{R}$ is meaningless).

As a byproduct of (77), one obtains (cf. [1], or (9.178) in [10] (p. 294)) the truncated Taylor approximation of the mass defect

$$
V(0) \rho_{0}-M=\frac{4 \pi r_{0}^{3} \rho_{0}}{3}\left[\frac{3}{10}\left(\frac{r_{0}}{\hat{R}}\right)^{2}+O\left(\frac{r_{0}}{\hat{R}}\right)^{4}\right]
$$

and uses (79) (together with the classical mechanics calculation of the surface potential on a sphere) to attribute $V(0) \rho_{0}-M$ to the loss of energy in packing the matter under its own gravitational energy. A similar formula for the mass defect $V(a) \rho_{a}-M$ is not known. That spherically symmetric solutions such as $\left\{g_{a}:-2 m<a<a_{1}\right\}\left[\right.$ where $a_{1}=\frac{9 r_{0}^{2}}{4}\left(r_{0}-2 m\right)-2 m$, cf. (51)] are appropriate for the calculation of mass defect of strange stars appears to be accounted for by [11].

Computing the bundle boundary (or Schmidt boundary, cf. [2]) of space-times $\left(\Omega, g_{a}\right),-2 m<$ $a<a_{1}$, and perhaps quantum-mechanically resolving the resulting singularities ([12-14]) is an open problem.

Author Contributions: The authors have equally contributed to the elaboration and writing of the paper.

Funding: This research received no external funding.

Conflicts of Interest: The authors declare no conflict of interest.

\section{Appendix A. An Abelian Integral}

Our purpose in this section is bringing to the canonical form the abelian integral

$$
\int \frac{x^{5 / 2} d x}{\sqrt{r_{0}^{3}-2 m \hat{R}^{2}+\hat{R}^{2} x-x^{3}}}=\int \mathcal{R}(x, \sqrt{P(x)}) d x, \quad P(x)=-x^{4}+\hat{R}^{2} x^{2}+\left(r_{0}^{3}-2 m \hat{R}^{2}\right) x,
$$

where $\mathcal{R}(x, y)$ is the rational function $\mathcal{R}(x, y)=x^{3} / y$. Starting from

$$
P(x)=-x\left(x^{3}+\hat{p} x+\hat{q}\right), \quad \hat{p}=-\hat{R}^{2}, \quad \hat{q}=2 m \hat{R}^{2}-r_{0}^{3},
$$


one sets as customary

$$
\left\{\lambda_{1}, \lambda_{2}\right\}=\left\{-\frac{\hat{q}}{2} \pm \sqrt{\frac{\hat{q}^{2}}{4}+\frac{\hat{p}^{3}}{27}}\right\}=\left\{\frac{1}{2}\left(r_{0}^{3}-2 m \hat{R}^{2}\right) \pm \sqrt{\frac{1}{4}\left(r_{0}^{3}-2 m \hat{R}^{2}\right)^{2}-\frac{\hat{R}^{6}}{27}}\right\}
$$

and distinguishes three cases as (I) $a^{2}>\frac{4}{27} \hat{R}^{2}$, (II) $a^{2}<\frac{4}{27} \hat{R}^{2}$, (III) $a^{2}=\frac{4}{27} \hat{R}^{2}$, where $a=\frac{r_{0}^{3}}{\hat{R}^{2}}-2 m$. Integral (A1) is abelian in Cases (I)-(II) (while in Case (III) it reduces to a rational integral). To apply the classical scheme of reduction to the canonical (Legendre) form, one needs a decomposition of (A2) into second-degree factors $P(x)=\left(x^{2}+p x+q\right)\left(x^{2}+p^{\prime} x+q^{\prime}\right)$. The required algebraic information is summarised in Table A1.

Table A1. Algebraic information on $P_{a}(x)$.

\begin{tabular}{|c|c|c|c|c|}
\hline Cases & $\lambda_{1}, \lambda_{2}$ & $P_{a}(x)$ & Real Root & Free Term \\
\hline (I) & & & & \\
\hline$a>0$ & $\begin{array}{l}\lambda_{1}>0 \\
\lambda_{2}>0\end{array}$ & $\begin{array}{c}(x-\sigma) \times \\
\left(x^{2}+\sigma x+\tau\right)\end{array}$ & $\begin{array}{l}\sigma \in \mathbb{R} \\
\sigma \neq 0\end{array}$ & $\tau>0$ \\
\hline$a<0$ & $\begin{array}{l}\lambda_{1}<0 \\
\lambda_{2}<0\end{array}$ & $\begin{array}{c}\left(x-\omega_{1} \sigma\right) \times \\
\left(x^{2}+\omega_{1} \sigma+\omega_{2} \tau\right)\end{array}$ & $\begin{array}{c}\omega_{1} \sigma \in \mathbb{R} \\
\sigma \neq 0\end{array}$ & $\omega_{2} \tau>0$ \\
\hline (II) & $\begin{array}{c}\lambda_{j} \in \mathbb{C} \backslash \mathbb{R} \\
\lambda_{2}=\bar{\lambda}_{1}\end{array}$ & $\begin{array}{c}\left(x-\omega_{1} \sigma\right) \times \\
\left(x^{2}+\omega_{1} \sigma+\omega_{2} \tau\right)\end{array}$ & $\omega_{1} \sigma \in \mathbb{R}$ & $\omega_{2} \tau \in \mathbb{R}$ \\
\hline
\end{tabular}

where $\sigma=\sqrt[3]{\lambda_{1}}+\sqrt[3]{\lambda_{2}}$ and $\tau=\left(\sqrt[3]{\lambda_{1}}\right)^{2}-\sqrt[3]{\lambda_{1}} \sqrt[3]{\lambda_{2}}+\left(\sqrt[3]{\lambda_{2}}\right)^{2}$. For the remainder of Appendix , our calculations are confined to the case $a>\frac{2}{3 \sqrt{3}} \hat{R}$. One starts from the decomposition $P(x)=$ $-\left(x^{2}-\sigma x\right)\left(x^{2}+\sigma x+\tau\right)$ and applies the homography $x=\frac{\mu t+v}{t+1}$ with $\mu, v \in \mathbb{R}$ and $\mu \neq v$, so that to obtain

$$
\begin{gathered}
x^{2}-\sigma x=\frac{1}{(t+1)^{2}}\left\{\mu(\mu-\sigma) t^{2}+[2 \mu \nu-\sigma(\mu+v)] t+v^{2}-\sigma \nu\right\}, \\
x^{2}+\sigma x+\tau=\frac{1}{(t+1)^{2}}\left\{\left(\mu^{2}+\sigma \mu+\tau\right) t^{2}+[\sigma(\mu+v)+2 \mu \nu+2 \tau] t+v^{2}+\sigma v+\tau\right\},
\end{gathered}
$$

together with the requirements $2 \mu \nu-\sigma(\mu+v)=0$ and $2 \mu \nu+\sigma(\mu+v)+2 \tau=0$ or equivalently $\mu+v=-\frac{\tau}{\sigma}$ and $\mu \nu=-\frac{\tau}{2}$ i.e.,

$$
\{\mu, v\}=\left\{\frac{1}{2 \sigma}\left[-\tau \pm \sqrt{\tau\left(\tau+2 \sigma^{2}\right)}\right]\right\}
$$

yielding

$$
\begin{gathered}
P(x)=-\frac{1}{(t+1)^{4}}\left(\mathcal{M}+\mathcal{N} t^{2}\right)\left(\mathcal{M}^{\prime}+\mathcal{N}^{\prime} t^{2}\right)=\frac{\mathcal{A}}{(t+1)^{4}}\left(1+\mathbf{m} t^{2}\right)\left(1+\mathbf{m}^{\prime} t^{2}\right), \\
\mathcal{M}=\frac{\tau}{2}-\left(\sigma+\frac{\tau}{\sigma}\right) v, \quad \mathcal{N}=\frac{\tau}{2}-\left(\sigma+\frac{\tau}{\sigma}\right) \mu, \\
\mathcal{M}^{\prime}=\frac{3 \tau}{2}+\left(\sigma-\frac{\tau}{\sigma}\right) v, \quad \mathcal{N}^{\prime}=\frac{3 \tau}{2}+\left(\sigma-\frac{\tau}{\sigma}\right) \mu, \\
\mathcal{A}=-\mathcal{M} \mathcal{M}^{\prime}, \quad \mathbf{m}=\frac{\mathcal{N}}{\mathcal{M}}, \quad \mathbf{m}^{\prime}=\frac{\mathcal{N}^{\prime}}{\mathcal{M}^{\prime}}
\end{gathered}
$$


So far,

$$
\begin{gathered}
\int \frac{x^{3} d x}{\sqrt{P(x)}}=\int \frac{\mathcal{R}^{*}(t) d t}{\sqrt{\mathcal{A}\left(1+\mathbf{m} t^{2}\right)\left(1+\mathbf{m}^{\prime} t^{2}\right)}}, \\
\mathcal{R}^{*}(t)=\frac{(v-\mu)(\mu t+v)^{3}}{[(\mu-\sigma) t+v-\sigma]\left(\mathcal{M}^{\prime}+\mathcal{N}^{\prime} t^{2}\right)},
\end{gathered}
$$

(where $\mu, v$ are given by (A3)). Note that

$$
\mathbf{m}=-\left(\frac{\mu}{v}\right)^{2}, \quad \mathbf{m}^{\prime}=\frac{\mu\left(\mu \nu-2 v^{2}+\mu^{2}\right)}{v\left(\mu \nu+v^{2}-2 \mu^{2}\right)} .
$$

The signs of the numbers $\mathbf{m}, \mathbf{m}^{\prime}$ and $\mathcal{A}$ are relevant (to the reduction to the canonical form).Note that $\mathcal{M}+\mathcal{N}=\frac{\tau^{2}}{\sigma^{2}}+2 \tau>0$ and $\mathcal{M N}=-\frac{\tau}{2}\left(\sigma^{2}+\frac{\tau}{2}\right)<0$ as $\tau>0$. Hence, $\mathbf{m}<0$. Moreover

$$
\mathcal{M}^{\prime}+\mathcal{N}^{\prime}=\frac{\tau^{2}}{\sigma^{2}}+2 \tau, \quad \mathcal{M}^{\prime} \mathcal{N}^{\prime}=\frac{\tau}{2}\left(\frac{2 \tau^{2}}{\sigma^{2}}-\sigma^{2}+\frac{7 \tau}{2}\right), \quad \mathbf{m}^{\prime}=\frac{\mathcal{N}^{\prime}}{\mathcal{M}^{\prime}}=\frac{3 \sigma \tau+2\left(\sigma^{2}-\tau\right) \mu}{3 \sigma \tau+2\left(\sigma^{2}-\tau\right) \nu}
$$

The discussion of the signs of $\mathbf{m}^{\prime}$ and $\mathcal{A}$ requires a disjunction of cases, as (i) $\sigma>0$ and $\tau<\sigma^{2}$, (ii) $\sigma>0$ and $\tau>\sigma^{2}$, (iii) $\sigma<0$ and $\tau<\sigma^{2}$, and (iv) $\sigma<0$ and $\tau>\sigma^{2}$. For instance, in Case (i) (by (A3)), $\mu>0>v$ and $3 \sigma \tau+2\left(\sigma^{2}-\tau\right) \mu>0$, while $3 \tau \sigma+2\left(\sigma^{2}-\tau\right) v>0 \Longleftrightarrow \sigma^{2}<4 \tau$, so that a further splitting of Case (i) in subcases is needed, as (i) $)_{1} \sigma>0$ and $\tau<\sigma^{2}<4 \tau$ or (i) $)_{2} \sigma>0$ and $\sigma^{2}>4 \tau$. In the end,

(i) $1 \quad \sigma>0, \tau<\sigma^{2}<4 \tau \Longrightarrow \mathbf{m}^{\prime}>0, \mathcal{A}<0$,

(i) $)_{2} \quad \sigma>0, \sigma^{2}>4 \tau \Longrightarrow \mathbf{m}^{\prime}<0, \mathcal{A}>0$,

(ii) $\sigma>0, \tau>\sigma^{2} \Longrightarrow \mathbf{m}^{\prime}>0, \mathcal{A}<0$,

(iii) $1 \quad \sigma<0, \tau<\sigma^{2}<4 \tau \Longrightarrow \mathbf{m}^{\prime}>0, \mathcal{A}<0$,

(iii) $)_{2} \quad \sigma<0, \sigma^{2}>4 \tau \Longrightarrow \mathbf{m}^{\prime}<0, \mathcal{A}>0$.

(iv) $\sigma<0, \tau>\sigma^{2} \Longrightarrow \mathbf{m}^{\prime}>0, \mathcal{A}<0$.

Next, we need to split (A5) into even and odd terms, i.e.,

$$
\mathcal{R}^{*}(t)=\frac{1}{2}\left[\mathcal{R}^{*}(t)+\mathcal{R}^{*}(-t)\right]+\frac{1}{2}\left[\mathcal{R}^{*}(t)-\mathcal{R}^{*}(-t)\right]=\mathcal{R}_{1}\left(t^{2}\right)+t \mathcal{R}_{2}\left(t^{2}\right)
$$

with

$$
\begin{aligned}
& \mathcal{R}_{1}(x)=(v-\mu) \frac{\mu(\mu-\sigma) x\left(\mu^{2} x+3 v^{2}\right)-v(v-\sigma)\left(3 \mu^{2} x+v^{2}\right)}{\left(\mathcal{M}^{\prime}+\mathcal{N}^{\prime} x\right)\left[(\mu-\sigma)^{2} x-(v-\sigma)^{2}\right]}, \\
& \mathcal{R}_{2}(x)=(v-\mu) \frac{v(\mu-\sigma)\left(v^{2}+3 \mu^{2} x\right)-\mu(v-\sigma)\left(3 v^{2}+\mu^{2} x\right)}{\left(\mathcal{M}^{\prime}+\mathcal{N}^{\prime} x\right)\left[(\mu-\sigma)^{2} x-(v-\sigma)^{2}\right]}
\end{aligned}
$$

so that (A4) becomes

$$
\int \frac{\mathcal{R}_{1}\left(t^{2}\right) d t}{\sqrt{\mathcal{A}\left(1+\mathbf{m} t^{2}\right)\left(1+\mathbf{m}^{\prime} t^{2}\right)}}+\frac{1}{2} \int \frac{\mathcal{R}_{2}(u) d u}{\sqrt{\mathcal{A}(1+\mathbf{m} u)\left(1+\mathbf{m}^{\prime} u\right)}}
$$

where the first integral should be further reduced to the canonical form, while the second is an ordinary Euler integral. Next, note that

$$
\mathcal{M}=-\frac{v^{2}(\mu-v)}{\mu+v}, \quad \mathcal{N}=\frac{\mu^{2}(\mu-v)}{\mu+v},
$$




$$
\begin{gathered}
\mathcal{M}^{\prime}=-\frac{v(\mu-v)(2 \mu+v)}{\mu+v}, \quad \mathcal{N}^{\prime}=\frac{\mu(\mu-v)(\mu+2 v)}{\mu+v}, \\
\mathcal{R}_{2}(u)=\frac{4 \mu v(\mu+v)(\mathcal{M}-\mathcal{N} u)}{(\mathcal{M}+\mathcal{N} u)\left(\mathcal{M}^{\prime}+\mathcal{N}^{\prime} u\right)} .
\end{gathered}
$$

Indeed, (A12) follows from

$$
\begin{gathered}
\mu^{2}[2 \mu v+\sigma(\mu-3 v)]=4 \mu v \mathcal{N}, \quad v^{2}[-2 \mu v+\sigma(3 \mu-v)]=-4 \mu v \mathcal{M}, \\
(\mu-\sigma)^{2}=\frac{\mu-v}{\mu+v} \mathcal{N}, \quad(v-\sigma)^{2}=-\frac{\mu-v}{\mu+v} \mathcal{M} .
\end{gathered}
$$

Q.e.d.

We only illustrate the treatment of Case (i) $)_{1}$ where $\mathbf{m}<0, \mathbf{m}^{\prime}>0$ and $\mathcal{A}<0$. For the Euler integral, Euler's substitution of the third kind is

$$
\sqrt{\mathcal{A}(1+\mathbf{m} u)\left(1+\mathbf{m}^{\prime} u\right)}=s\left(u+\frac{1}{\mathbf{m}^{\prime}}\right), \quad u \in \mathbb{R} \backslash\left[-\frac{1}{\mathbf{m}^{\prime}},-\frac{1}{\mathbf{m}}\right] .
$$

Hence,

$$
\begin{gathered}
u=\frac{s^{2}-\mathbf{m}^{\prime 2} \mathcal{A}}{\mathbf{m}^{\prime}\left(\mathbf{m m}^{\prime} \mathcal{A}-s^{2}\right)}, \quad s^{2}=\mathbf{m}^{\prime 2} \mathcal{A} \frac{1+\mathbf{m} u}{1+\mathbf{m}^{\prime} u}, \\
d u=\frac{2\left(\mathbf{m}-\mathbf{m}^{\prime}\right) \mathcal{A} s}{\left(\mathbf{m m}^{\prime} \mathcal{A}-s^{2}\right)^{2}} d s, \sqrt{\mathcal{A}(1+\mathbf{m} u)\left(1+\mathbf{m}^{\prime} u\right)}=\frac{\left(\mathbf{m}-\mathbf{m}^{\prime}\right) \mathcal{A} s}{\mathbf{m}^{\prime} \mathcal{A}-s^{2}},
\end{gathered}
$$

and [by (A8) with $\left.x=\frac{s^{2}-\mathbf{m}^{\prime 2} \mathcal{A}}{\mathbf{m}^{\prime}\left(\mathbf{m m}^{\prime} \mathcal{A}-s^{2}\right)}\right]$

$$
\mathcal{R}_{2}\left(\frac{s^{2}-\mathbf{m}^{\prime 2} \mathcal{A}}{\mathbf{m}^{\prime}\left(\mathbf{m m}^{\prime} \mathcal{A}-s^{2}\right)}\right)=\frac{4 \mu(\mu+v)^{2}}{(v-\mu)(2 \mu+v)} \frac{\left(\mathcal{A} \mathbf{m} \mathbf{m}^{\prime}-s^{2}\right)\left[2 \mathcal{A} \mathbf{m} \mathbf{m}^{\prime 2}-s^{2}\left(\mathbf{m}+\mathbf{m}^{\prime}\right)\right]}{\mathbf{m}^{\prime} \mathcal{A}\left(\mathbf{m}-\mathbf{m}^{\prime}\right)^{2} s^{2}}
$$

so that

$$
\frac{1}{2} \int \frac{\mathcal{R}_{2}(u) d u}{\sqrt{\mathcal{A}(1+\mathbf{m} u)\left(1+\mathbf{m}^{\prime} u\right)}}=\frac{4 \mu(\mu+v)^{2}}{(\mu-v)(2 \mu+v)} \frac{1}{\left(\mathbf{m}-\mathbf{m}^{\prime}\right)^{2}}\left[\frac{2 \mathbf{m ~ m}^{\prime}}{s}+\frac{\left(\mathbf{m}+\mathbf{m}^{\prime}\right) s}{\mathbf{m}^{\prime} \mathcal{A}}\right]
$$

or

$$
\frac{1}{2} \int \frac{\mathcal{R}_{2}(u) d u}{\sqrt{\mathcal{A}(1+\mathbf{m} u)\left(1+\mathbf{m}^{\prime} u\right)}}=\mathcal{S}(u)
$$

where

$$
\begin{gathered}
\mathcal{S}(u):=\frac{4 \mu(\mu+v)^{2}}{(\mu-v)(2 \mu+v)} \frac{1}{\left(\mathbf{m}-\mathbf{m}^{\prime}\right)^{2}} \times \\
\times\left[\frac{2 \mathbf{m}\left(1+\mathbf{m}^{\prime} u\right)}{\sqrt{\mathcal{A}(1+\mathbf{m} u)\left(1+\mathbf{m}^{\prime} u\right)}}+\left(\mathbf{m}+\mathbf{m}^{\prime}\right) \frac{\sqrt{\mathcal{A}(1+\mathbf{m} u)\left(1+\mathbf{m}^{\prime} u\right)}}{\mathcal{A}\left(1+\mathbf{m}^{\prime} u\right)}\right] .
\end{gathered}
$$

Going back to (A9), we set $\mathbf{m}=-h^{2}$ and $\mathbf{m}^{\prime}=h^{\prime 2}$ (with $h>0$ and $h^{\prime}>0$ ). Let us substitute from

$$
h t=\frac{1}{\sqrt{1-z^{2}}}, \quad h d t=\frac{z d z}{\left(1-z^{2}\right)^{3 / 2}}
$$




$$
\begin{gathered}
\frac{d t}{\sqrt{\mathcal{A}\left(1-h^{2} t^{2}\right)\left(1+{h^{\prime 2}}^{2}\right)}}=\frac{1}{\sqrt{-\mathcal{A}\left(h^{2}+{h^{\prime}}^{2}\right)}} \frac{d z}{\sqrt{\left(1-z^{2}\right)\left(1-k^{2} z^{2}\right)}}, \quad k:=\frac{h}{\sqrt{h^{2}+h^{\prime 2}}}, \\
\mathcal{R}_{1}\left(t^{2}\right)=(\mu+v) \mathcal{R}_{1}^{*}\left(z^{2}\right), \quad \mathcal{R}_{1}^{*}(x):=\frac{\mu^{4}+6 \mu^{2} v^{2} h^{2}(1-x)+v^{4} h^{4}(1-x)^{2}}{\left[h^{2}(1-x) v^{2}-\mu^{2}\right]\left[h^{2}(1-x) \mathcal{M}^{\prime}+\mathcal{N}^{\prime}\right]},
\end{gathered}
$$

into (A9), so that to obtain

$$
\int \frac{\mathcal{R}_{1}\left(t^{2}\right) d t}{\sqrt{\mathcal{A}\left(1-h^{2} t^{2}\right)\left(1+h^{\prime 2} t^{2}\right)}}=\frac{\mu+v}{\sqrt{-\mathcal{A}\left(h^{2}+h^{\prime 2}\right)}} \int \frac{\mathcal{R}_{1}^{*}\left(z^{2}\right) d z}{\sqrt{\left(1-z^{2}\right)\left(1-k^{2} z^{2}\right)}} .
$$

Note that (by (A6)) $h^{2}=\mu^{2} / v^{2}$, and a calculation shows that

$$
\mathcal{R}_{1}^{*}(x)=\frac{v^{2}}{\mathcal{M}^{\prime}}\left\{1-\frac{8 k^{2}}{x}+\left[8\left(k^{2}-1\right)+\frac{1}{k^{2}}\right] \frac{1}{x-\frac{1}{k^{2}}}\right\} .
$$

If we adopt the customary notations

$$
\begin{gathered}
I_{n}=\int \frac{z^{2 n} d z}{\sqrt{\left(1-z^{2}\right)\left(1-k^{2} z^{2}\right)}}, \quad n \in \mathbb{Z}_{+}, \\
H_{m}(\alpha)=\int \frac{d z}{\left(z^{2}-\alpha\right)^{m} \sqrt{\left(1-z^{2}\right)\left(1-k^{2} z^{2}\right)}}, \quad m \in \mathbb{N}, \quad \alpha \in \mathbb{R},
\end{gathered}
$$

then (by (A15))

$$
\int \frac{\mathcal{R}_{1}^{*}\left(z^{2}\right) d z}{\sqrt{\left(1-z^{2}\right)\left(1-k^{2} z^{2}\right)}}=\frac{v^{2}}{\mathcal{M}^{\prime}}\left\{I_{0}-8 k^{2} H_{1}(0)+\left[8\left(k^{2}-1\right)+\frac{1}{k^{2}}\right] H_{1}\left(\frac{1}{k^{2}}\right)\right\}
$$

where

$$
I_{0}=\int \frac{d z}{\sqrt{\left(1-z^{2}\right)\left(1-k^{2} z^{2}\right)}}=\int \frac{d \phi}{\sqrt{1-k^{2} \sin ^{2} \phi}}
$$

(with $z=\sin \phi, 0<\phi<\pi / 2)$ is certainly an elliptic integral of the first kind. As to $H_{1}(0)$, one starts from the (well-known) recurrence relation

$$
\begin{gathered}
(2 m-2)\left[-\alpha+\left(k^{2}+1\right) \alpha^{2}-k^{2} \alpha^{3}\right] H_{m}(\alpha)-(2 m-3)\left[1-2 \alpha\left(k^{2}+1\right)+3 k^{2} \alpha^{2}\right] H_{m-1}(\alpha)+ \\
+(2 m-4)\left(k^{2}+1-3 k^{2} \alpha\right) H_{m-2}(\alpha)-(2 m-5) k^{2} H_{m-3}(\alpha)=\frac{z}{\left(z^{2}-\alpha\right)^{m-1}} \sqrt{\left(1-z^{2}\right)\left(1-k^{2} z^{2}\right)}
\end{gathered}
$$

and sets $m=2$ and $\alpha=0$, so that to obtain

$$
-H_{1}(0)+k^{2} H_{-1}(0)=\frac{1}{z} \sqrt{\left(1-z^{2}\right)\left(1-k^{2} z^{2}\right)}
$$

and $H_{-1}(0)=I_{1}$, where

$$
I_{1}=\int \frac{z^{2} d z}{\sqrt{\left(1-z^{2}\right)\left(1-k^{2} z^{2}\right)}}=\int \frac{\sin ^{2} \phi d \phi}{\sqrt{\left(1-z^{2}\right)\left(1-k^{2} z^{2}\right)}}
$$




$$
=\frac{1}{k^{2}} \int \frac{d \phi}{\sqrt{1-k^{2} \sin ^{2} \phi}}-\frac{1}{k^{2}} \int \sqrt{1-k^{2} \sin ^{2} \phi} d \phi
$$

is certainly an elliptic integral of the second kind. Elliptic integrals of the third kind

$$
\int \frac{d z}{\left(1+\beta z^{2}\right) \sqrt{\left(1-z^{2}\right)\left(1-k^{2} z^{2}\right)}}, \quad 0<k<1, \quad \beta \in \mathbb{C},
$$

may be derived from $H_{1}(\alpha)$ with $\alpha \neq 0$ by setting $\beta=-1 / \alpha$; hence,

$$
\begin{gathered}
H_{1}\left(\frac{1}{k^{2}}\right)=-k^{2} \int \frac{d z}{\left(1-k^{2} z^{2}\right) \sqrt{\left(1-z^{2}\right)\left(1-k^{2} z^{2}\right)}} \\
=-k^{2} \int \frac{d \phi}{\left(1-k^{2} \sin ^{2} \phi\right) \sqrt{1-k^{2} \sin ^{2} \phi}} .
\end{gathered}
$$

Consequently, (by (A17)-(A19)), Equation (A16) becomes

$$
\begin{aligned}
& \int \frac{\mathcal{R}_{1}^{*}\left(z^{2}\right) d z}{\sqrt{\left(1-z^{2}\right)\left(1-k^{2} z^{2}\right)}}=\frac{v^{2}}{\mathcal{M}^{\prime}}\left\{8 k^{2} \cot \phi \sqrt{1-k^{2} \sin ^{2} \phi}\right. \\
& +\left(1-8 k^{2}\right) \int \frac{d \phi}{\sqrt{1-k^{2} \sin ^{2} \phi}}+8 k^{2} \int \sqrt{1-k^{2} \sin ^{2} \phi} d \phi \\
& \left.+\left[8 k^{2}\left(1-k^{2}\right)-1\right] \int \frac{d \phi}{\left(1-k^{2} \sin ^{2} \phi\right) \sqrt{1-k^{2} \sin ^{2} \phi}}\right\} .
\end{aligned}
$$

Summing up (by (A1), (A4), (A9), (A13), (A14) and (A20)) under the transformations

$$
x=\frac{\mu t+v}{t+1}, \quad h t=\frac{1}{\sqrt{1-z^{2}}}, \quad z=\sin \phi,
$$

(A1) becomes

$$
\begin{aligned}
& \int \frac{x^{5 / 2} d x}{\sqrt{r_{0}^{3}-2 m \hat{R}^{2}+\hat{R}^{2} x-x^{3}}}=\frac{k v^{2}(\mu+v)}{h \mathcal{M}^{\prime} \sqrt{-\mathcal{A}}}\left\{8 k^{2} \cot \phi \sqrt{1-k^{2} \sin ^{2} \phi}\right. \\
& +\left(1-8 k^{2}\right) \int \frac{d \phi}{\sqrt{1-k^{2} \sin ^{2} \phi}}+8 k^{2} \int \sqrt{1-k^{2} \sin ^{2} \phi} d \phi \\
& \left.+\left[8 k^{2}\left(1-k^{2}\right)-1\right] \int \frac{d \phi}{\left(1-k^{2} \sin ^{2} \phi\right) \sqrt{1-k^{2} \sin ^{2} \phi}}\right\}+\mathcal{S}\left(\frac{1}{h^{2} \cos ^{2} \phi}\right)
\end{aligned}
$$

where

$$
\mathcal{S}\left(\frac{1}{h^{2} \cos ^{2} \phi}\right)=\frac{4 k^{3} \mu(\mu+v)^{2}}{h^{2}(v-\mu)(2 \mu+v) \sqrt{-\mathcal{A}}} \frac{2+\left(1-4 k^{2}\right) \sin ^{2} \phi}{\sin \phi \sqrt{1-k^{2} \sin ^{2} \phi}} .
$$


Note that (by (A11)) $\frac{v^{2}(\mu+v)}{\mathcal{M}^{\prime}}=\frac{v(\mu+v)^{2}}{(v-\mu)(2 \mu+v)}$; hence, the "divergent" terms in (A21) sum up to (by $v h+\mu=0$ )

$$
\frac{4 k^{3}(\mu+v)^{2} \mu}{h^{2}(v-\mu)(2 \mu+v) \sqrt{-\mathcal{A}}} \frac{1}{\sin \phi}\left[\frac{2+\left(1-4 k^{2}\right) \sin ^{2} \phi}{\sqrt{1-k^{2} \sin ^{2} \phi}}-2 \cos \phi \sqrt{1-k^{2} \sin ^{2} \phi}\right] .
$$

\section{References}

1. Schwarzschild, K. Über das Gravitationsfeld einer Kugel aus Inkompressibler Flüssigkeit nach der Einsteinschen Theorie; Sitzber. Preuss. Akad. Wiss.: Berlin, Germany, 1916; pp. 424-434.

2. Clarke, C.J.S. The Analysis of Space-Time Singularities; Cambridge University Press: Cambridge, UK, 1993.

3. Florides, P.S. A new interior Schwarzschild solution. Proc. Royal Soc. London Ser. A 1974, 337, 529-535.

4. Mehra, A.L. An interior solution for a charge sphere in general relativity. Phys. Lett. A 1983, 88, 159-161. [CrossRef]

5. Kofinti, N.K. On a new interior Schwarzschild solution. Gen. Relativ. Grav. 1985, 17, 245-249. [CrossRef]

6. Gron, O. A charged generalization of Florides' interior Schwarzschild solution. Gen. Relativ. Grav. 1986, 18, 591-596. [CrossRef]

7. Ségransan, D.; Kervella, P.; Forveille, T.; Queloz, D. First radius measurements of very low mass stars with the VLTI. Astron. Astrophys. 2003, 397, L5-L8. [CrossRef]

8. Carroll, W.B.; Ostlie, A.D. An Introduction to Modern Astrophysics; Cambridge University Press, University Printing House: Cambridge, UK, 2017; p. 409.

9. Ribas, I. Solar and Stellar Variability: Impact on Earth and Planets; Kosovichev, A.G., Andrei, A.H., Roelot, J.P., Eds.; International Astronomical Union Symposium, Volume 264; Cambridge University Press: Cambridge, UK, 2010.

10. Adler, R.; Bazin, M.; Schiffer, M. Introduction to General Relativity; McGraw-Hill Book Co.: New York, NY, USA, 1965.

11. Vartanyan, Y.L.; Grigoryan, A.K.; Khachatryan, G.A. On the mass defect of strange stars. Astrophysics 1995, 38, 152-156. [CrossRef]

12. Ashtekar, A.; Bojowald, M. Quantum geometry and the Schwarzschild singularity. arXiv 2005, arXiv:gr-qc/0509075v1.

13. Husain, V.; Winkler, O. On singularity resolution in quantum gravity. Phys. Rev. D 2003, 69, 084016. [CrossRef]

14. Husain, V:; Winkler, O. Quantum resolution of black hole singularities. Class. Quantum Gravity 2005, 22, L127. [CrossRef]

Publisher's Note: MDPI stays neutral with regard to jurisdictional claims in published maps and institutional affiliations.

(C) 2020 by the authors. Licensee MDPI, Basel, Switzerland. This article is an open access article distributed under the terms and conditions of the Creative Commons Attribution (CC BY) license (http:// creativecommons.org/licenses/by/4.0/). 